إنتاج وصادرات البطاطس المصرية ووضعها التنافسي في الأسواق العالمية

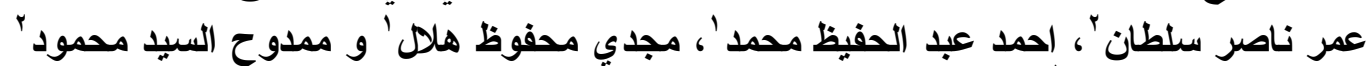

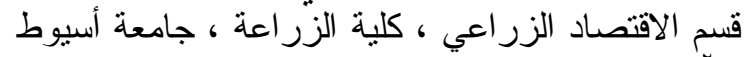

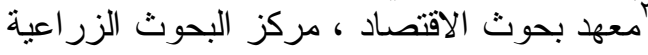

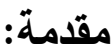

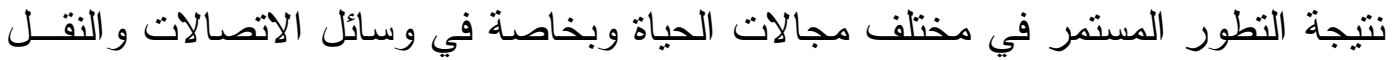

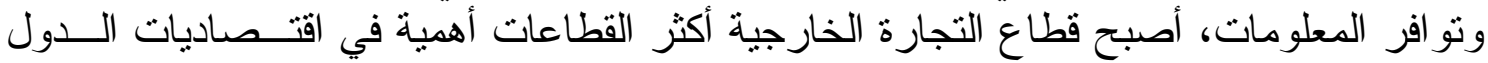

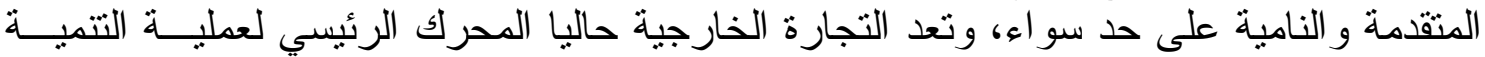

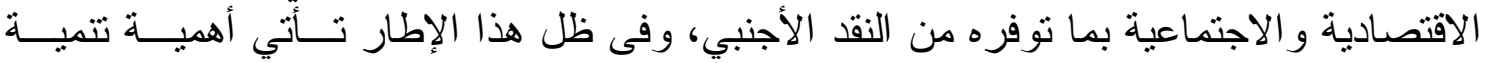

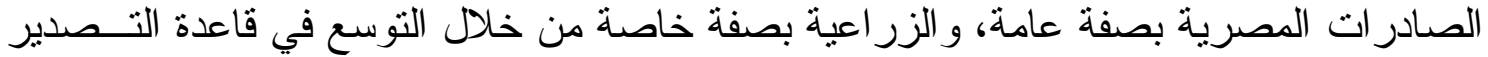

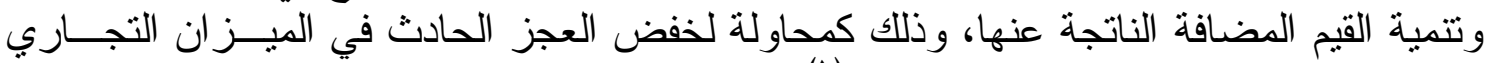

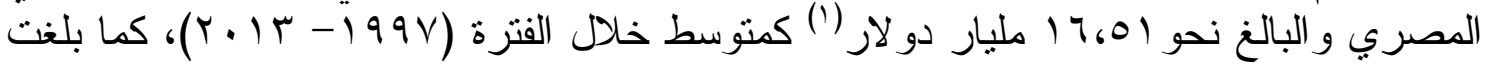

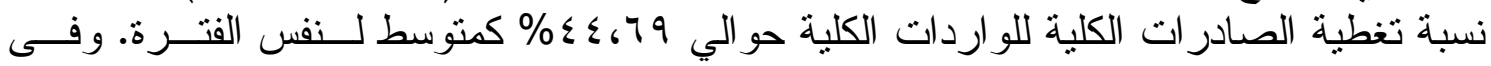

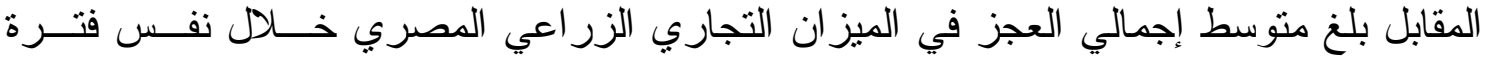

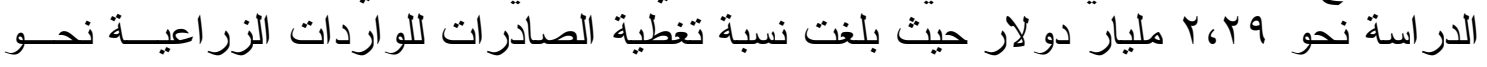

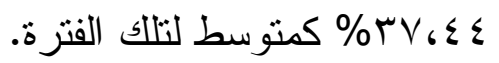

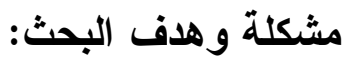

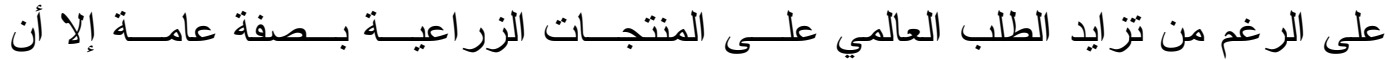

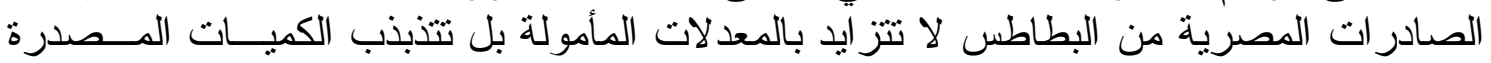

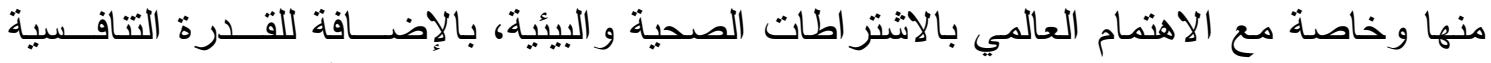

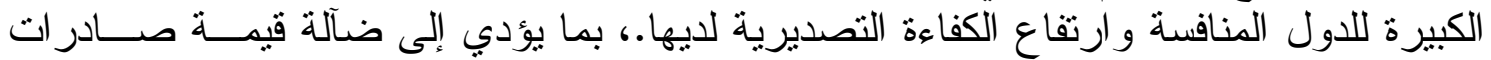

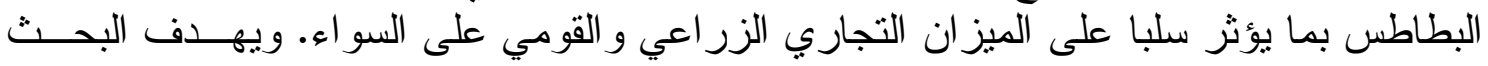

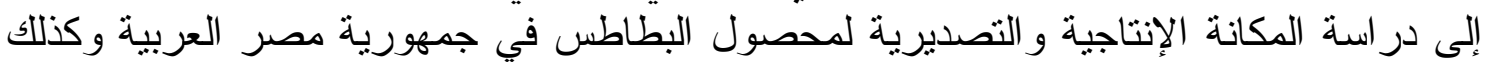

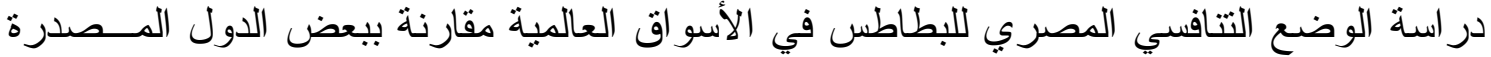

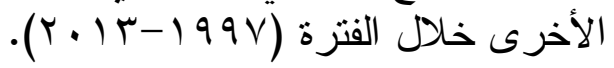
الطريقة البحثية ومصادر البيانات:

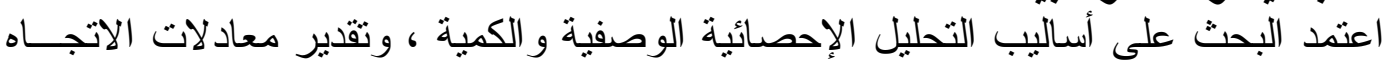

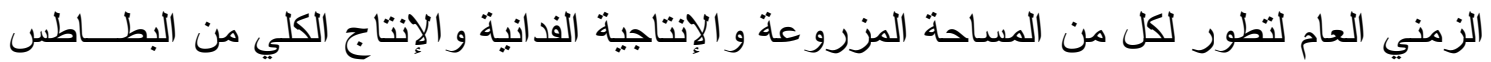

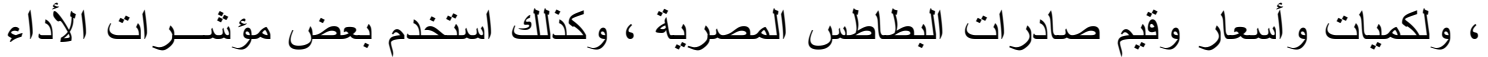

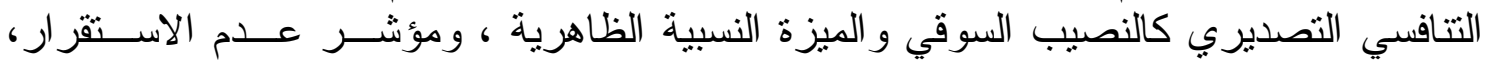

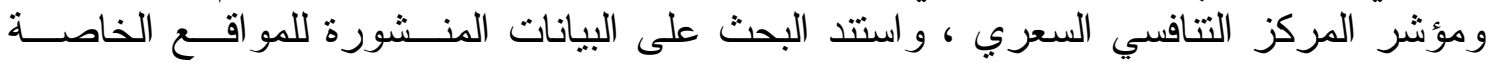

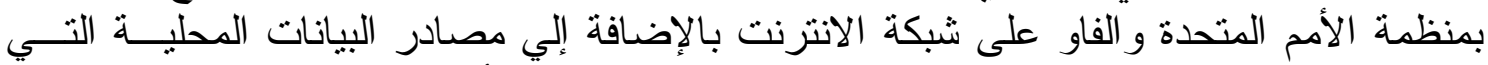

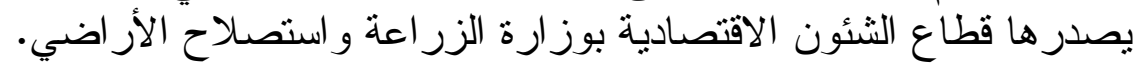

Received on: 3/2/2015

Referees: Prof. Salah S. Saleh
Accepted for publication on: 17/2/2015

Prof. El Metwaly S. Elzanaty 
اعتمد تحليل الأوضاع التتافسية في هذا البحث على مجموعة من المعايير المسـتخدمة (')

الإطار التحليلي:

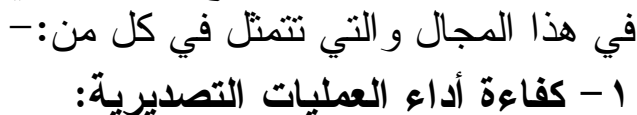

يمثل هذا المعيار أحد أهم المؤشرات العامة لقياس كفاءة النظم و الــسياسات و الهيئـات و المؤسسات التصديرية داخل الدولة ، وقدرتها على إنــــهاء الإجر اءات و التسهيلات التصديرية. ويتم تقدير هذا المعيار على النحو التالي:

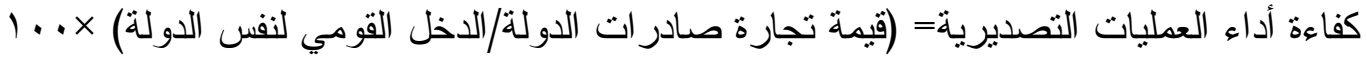

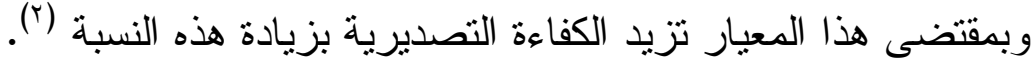
جدول رقم (1): الميزان التجاري القومي المصري والاخل القومي بالمليار دولار

\begin{tabular}{|c|c|c|c|c|c|c|}
\hline التصديرية الكفاعة & الاخل القومي & /واردات /صادرات & عجز الميزان & القومية & القومية الصادرات & السنوات \\
\hline$\varepsilon_{6 \vee V A}$ & NI.V7 & r9.71 & $\left(96 Y^{\prime}\right)$ & Ir.IV & $r .91$ & $199 V$ \\
\hline$r .70$ & $\Lambda V_{6}, 0 \Lambda$ & $196 \% 9$ & $\left(I T_{6} \mathrm{rA}\right)$ & $176 \leqslant 1$ & r.r. & 1991 \\
\hline r.V9 & QY.TY & Y1.9T & $\left(I_{1} Y_{6} \leqslant T\right)$ & 10,97 & r.0. & 1999 \\
\hline 8691 & 90,07 & rr.7I & $(9, Y V)$ & 11497 & 2679 & $r \ldots$ \\
\hline$\varepsilon .0 \mathrm{~V}$ & $9.60 \mathrm{~V}$ & rr.00 & $\left(\wedge_{6}, 0 \wedge\right)$ & IY.VT & 纟6) $\varepsilon$ & $r \ldots 1$ \\
\hline $0, \leqslant \leqslant$ & NT.VT & MVGT & $\left(V_{\triangleleft} \wedge \Lambda\right)$ & $1 T_{60}$ & E.77 & $r \ldots r$ \\
\hline$\Lambda_{6} \mathrm{TV}^{\mathrm{N}}$ & $V r_{609}$ & $07.0\}$ & $\left(\varepsilon_{6} V T\right)$ & 1.619 & 7.17 & $r \ldots r$ \\
\hline $9 . V V$ & $V \wedge_{6} 09$ & 09611 & $(0,17)$ & TY.AE & $V .71$ & $r \ldots \varepsilon$ \\
\hline 11.49 & $9 r_{6} \leqslant 0$ & Or.VT & $(9,1 \vee)$ & 19.11 & 1.670 & $r \ldots o$ \\
\hline $1 T_{6} / 9$ & $1 . V_{6} Y_{7}$ & 77.74 & $(7, \wedge \vee)$ & $r .609$ & IM.VT & $Y \ldots r$ \\
\hline $1 r_{6} \cdot 1$ & ITE $T$ I & 09611 & $(1 \cdot ، \wedge \top)$ & $r V_{6} \cdot r$ & 17.18 & $r \ldots V$ \\
\hline $10, \varepsilon \Lambda$ & $17 V_{6} V T$ & $\sum 9, Y T$ & $\left(Y^{\prime} \backslash \vee \wedge\right)$ & Or.VO & To.9V & $r \ldots \Lambda$ \\
\hline IY.VY & 19.617 & Or.AE & $(Y \cdot \sigma V T)$ & $\varepsilon \varepsilon(9)$ & $r \leqslant 61 \wedge$ & $r \ldots q$ \\
\hline IT.rT & Y)r. & $\leqslant 9.71$ & $\left(Y T^{\prime} 7 V\right)$ & or... & rT.rT & $r \cdot 1$. \\
\hline$I T_{G} V T$ & $r r \cdot . .$. & 0.671 & $\left(r \cdot{ }^{\prime} \cdot v \cdot\right)$ & TYGKA & 11.01 & 4.11 \\
\hline 11,71 & TOMGMY & $\varepsilon Y_{6} \| 1$ & $(\varepsilon \cdot ، \leqslant 0)$ & $79.1 \mathrm{~V}$ & $r q, \varepsilon r$ & $r .1 Y$ \\
\hline $1.6 \mathrm{TV}$ & r79.Ar & $\varepsilon r_{6} \mid V$ & $\left(r v_{6} \wedge q\right)$ & $77.7 V$ & rA.VA & $r .1 T$ \\
\hline $9 . r$. & $T \Gamma V_{6} 91$ & $\varepsilon \varepsilon 679$ & $(17.01)$ & $r .691$ & 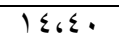 & المتوسط \\
\hline
\end{tabular}

المصدر : جمعت وحسبت من بيانات: ل Statistics database (www.comtrade.UN.Org

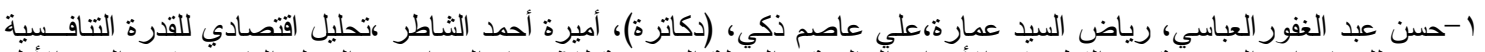

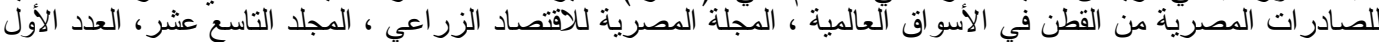

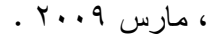

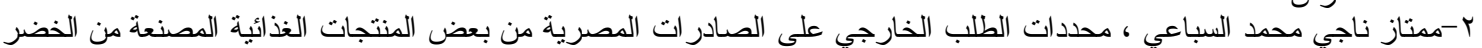

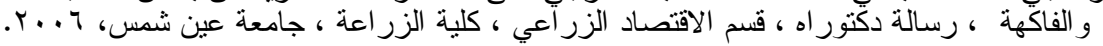




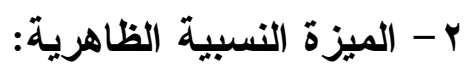

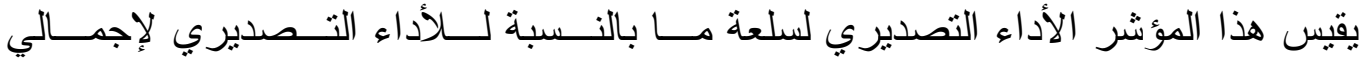
الصادرات على المستوي المحلي مقارنا بنفس النسبة على المستوي العالمي.

R.C. $\mathrm{A}=\left(\mathrm{E}_{\mathrm{IJ}} / E_{J}\right) /\left(\mathrm{E}_{\mathrm{IW}} / E_{W}\right)$

وينم تقديره على النحو التالي :

\author{
( الميزة النسبية الظاهرية اللسلعة الزر اعية ( I ) R.C.A

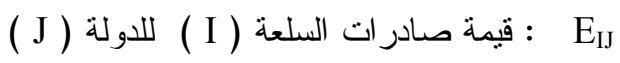

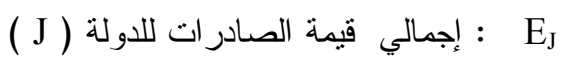

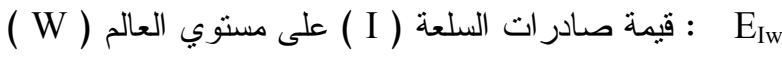

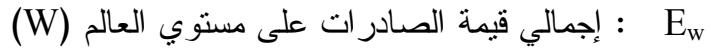

وطبقأ لهذا المعيار إذا كانت قيمة R.C.A اكبر من و احد صحيح دل ذلك على وجود ميزة

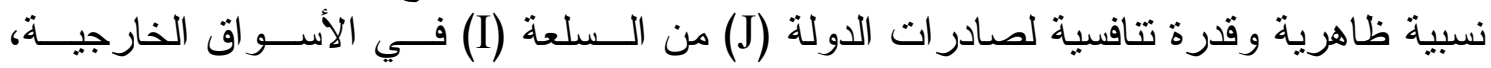

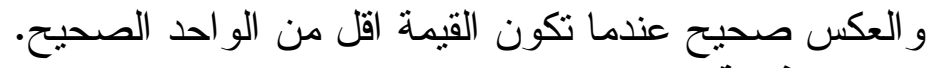

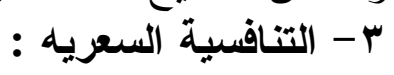

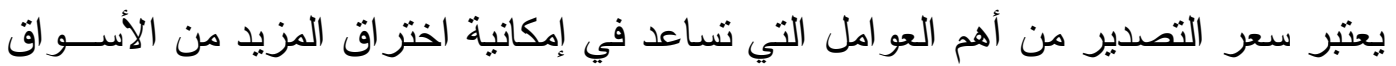

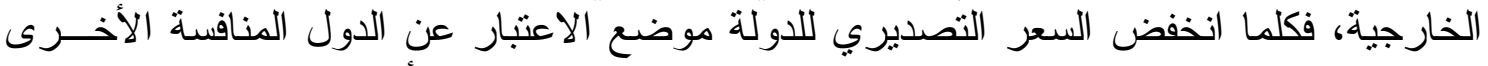

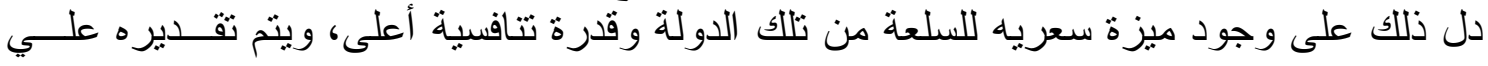

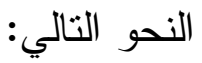
$P . C=P_{E} / P_{C}$

: معامل التنافسية السعريه . P.C

حيث:

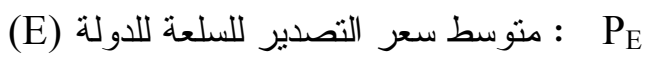

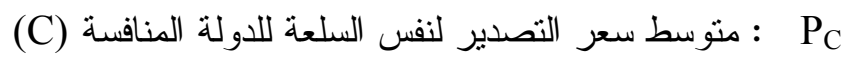

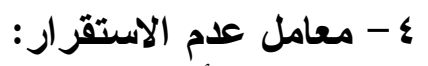

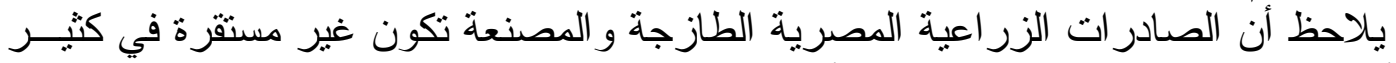

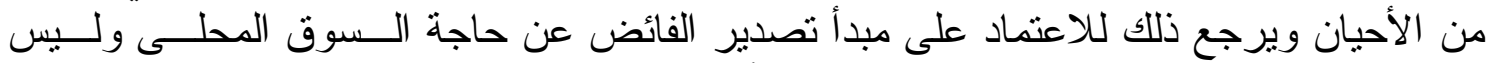

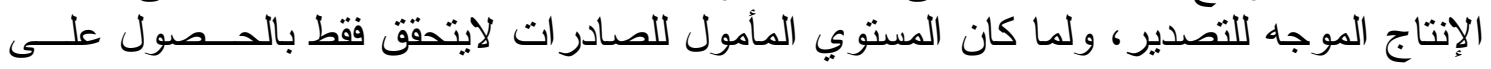

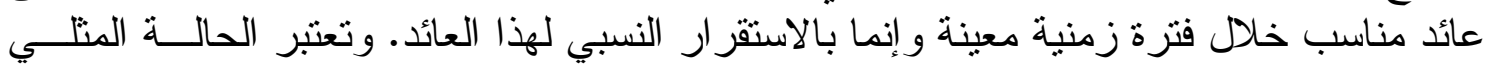

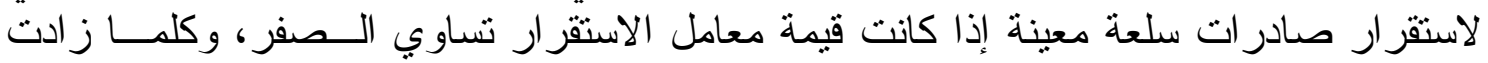

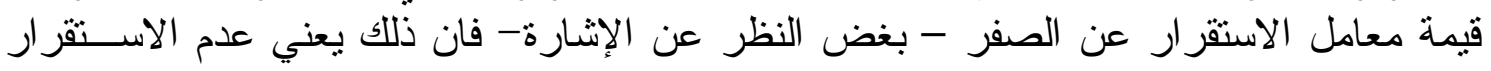
للصادر ات.وتقدر قيمة هذا المعامل علي النحو التالي:

$S . C=\left(\left(Y_{I}-\hat{Y}_{I}\right) /\left(\hat{Y}_{I}\right)\right) * 100$

حيث:

$$
\text { • : S.C }
$$

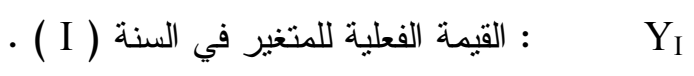




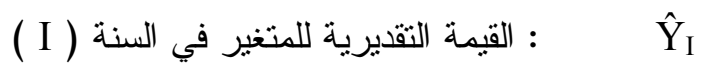

\section{ه - النصيب السوقي:}

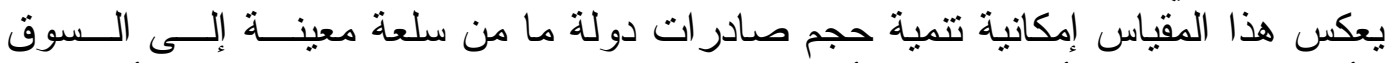

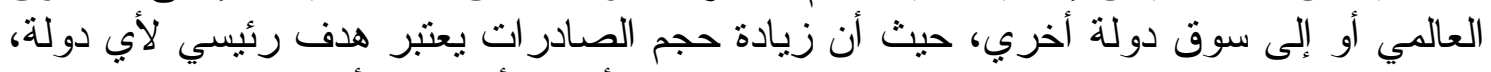

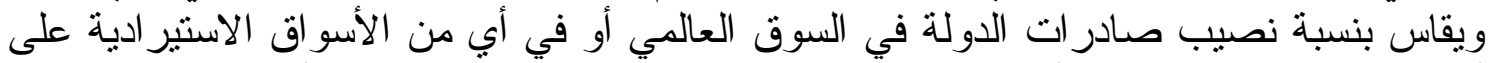

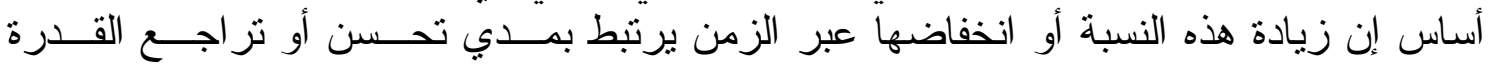
$M \cdot S_{J}=\left(X_{I J} / \sum_{J=1}^{N} X_{I J}\right) * 100$

التتافسية، ويقاس النصيب السوقي بالمعادلة التالية:

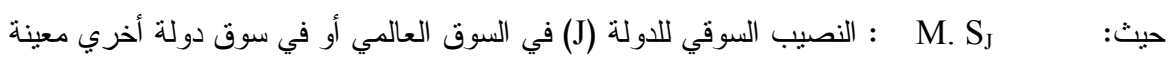

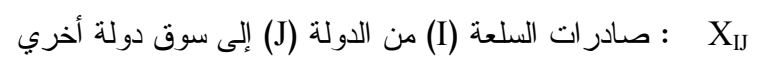

$$
\begin{aligned}
& \text { (أو إجمالي الواردات العالمية) : } \sum_{J=1}^{N} X_{J I}
\end{aligned}
$$

نتائج البحث:

أولا : المعالم الإنتاجية للبطاطس المصرية:

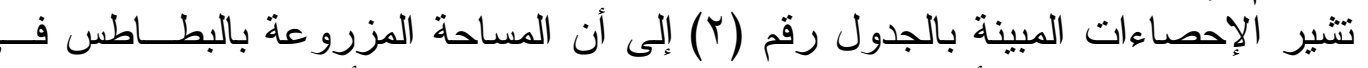

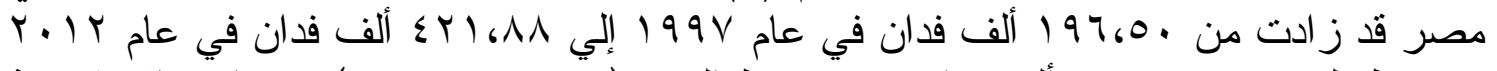

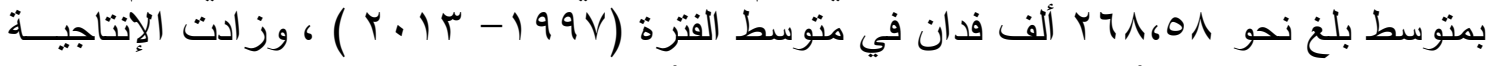

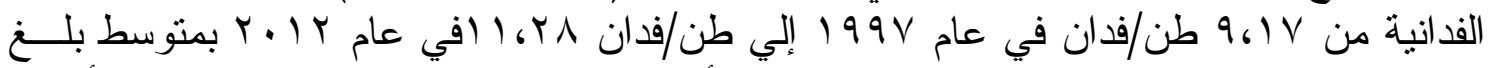

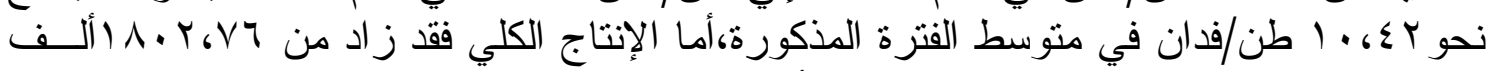

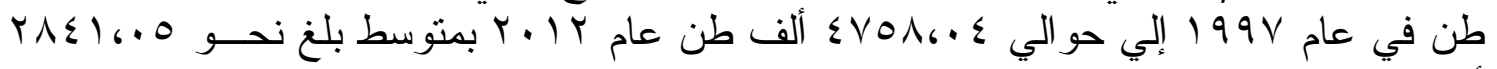
ألف طن لنف في عام الفترة. جدول رقم (Y): تطور المساحة المزروعة والإتتاجية الفدانية والإتتاج الكلي من البطاطس فـي

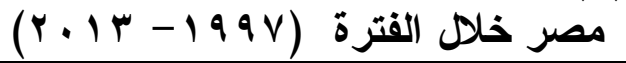

\begin{tabular}{|c|c|c|c|c|c|c|}
\hline الرقم القياسي & بالألفَ طن & الرقم القياسي & طن / الإتاجية & الرقم القياسي & بالألف فدانة & وات البيان \\
\hline $1 \ldots$ & $1 A \cdot Y_{6} V 4$ & $1 \ldots$ & $9.1 \mathrm{~V}$ & $1 \ldots$ & 19760 & $199 \mathrm{~V}$ \\
\hline 11.6 .0 & $19 \wedge \varepsilon_{6} \cdot 1$ & $1 . Y_{6} Y_{9}$ & $9.4 \mathrm{~N}$ & $1 . V(7)$ & rll، & 1991 \\
\hline 1... & $11 \cdot \Lambda_{6} \wedge 9$ & 1.7 .67 & 9.19 & $9 \varepsilon_{6} \cdot V$ & $1 \wedge \varepsilon ، \wedge \varepsilon$ & 1999 \\
\hline $9 V_{6} 9$. & $\mid V 7 \varepsilon 691$ & $1 \cdot V_{6} V \varepsilon$ & $96 \wedge 1$ & $9 \cdot 69 \leqslant$ & $1 \vee 1.79$ & r... \\
\hline $1.0 .0 \mathrm{~V}$ & 19.4 .14 & 1.9 .41 & 1.6 .4 & 79.0. & 119967 & $r \ldots l$ \\
\hline 11.614 & $1910,4 \mathrm{Yr}$ & $11.61 \varepsilon$ & 1.61. & $1 \ldots . \cdot v$ & $197.7 \leqslant$ & Y.. T \\
\hline $11 r_{6} 1 Y$ & $r .49, r_{0}$ & $11 Y_{6} V^{-}$ & $1.6 r \varepsilon$ & $1 \cdots, 4 \mathrm{r}$ & $19860_{0}$ & $r \ldots r$ \\
\hline $1 \leqslant 1,47$ & ros T.7T & 111.99 & $1.6 \mathrm{TV}$ & TYTGY & $r \leqslant \Lambda_{6} \cdot \varepsilon$ & $r \ldots \varepsilon$ \\
\hline IVOGV. & rITV،\&r & $11 \varepsilon_{\Delta, A T}$ & 1.604 & 1046.1 & $r \ldots .74$ & T..o \\
\hline TYA.rq & YMIYGVA & $11 \varepsilon_{6} 0$. & 1.60. & $11 r_{6} \cdot 7$ & KY.G. & $r \ldots \tau$ \\
\hline 104,15 & YV7.،\&T & 118.07 & $1.6 \mathrm{VA}$ & $1 \% \cdot 699$ & ro76. T & $r \ldots v$ \\
\hline $19 V_{6} \mathrm{AV}$ & rotV..0 & $111_{6} \times 7$ & 1.619 & 177.75 & TYV_EY & $r \ldots \lambda$ \\
\hline T.Y.9A & rro9,rA & $1 Y 16.0$ & $11,1$. & $17 V_{6} A$. & rYq.VY & $r \ldots q$ \\
\hline$r .1,09$ & rTTE,TY & $11 \Lambda_{6} \varepsilon r$ & $1 \cdot 617$ & $T V \cdot \sigma r \cdot$ & $T T \varepsilon, T \varepsilon$ & r.1. \\
\hline$T \leqslant \cdot 670$ & $\varepsilon r r \Lambda_{6} \varepsilon r$ & $1 Y 16.0$ & 1161. & 191619 & $r 9 \cdot 6 \wedge 1$ & $r .11$ \\
\hline Yqr.q. & $\varepsilon V_{0} \Lambda_{6} \cdot \varepsilon$ & TYr6. & 11,51 & Y) ह6. & $\sum r \mid, A \Lambda$ & $T \cdot I T$ \\
\hline rr4.09 & $\varepsilon r 70,11$ & TYI.9Y & 11,111 & $19 \varepsilon 6.9$ & rNI.rA & $T .1 T$ \\
\hline- & 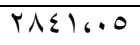 & - & $1 \cdot 6 \leqslant Y$ & - & $r 71.01$ & المتوسط \\
\hline
\end{tabular}


المصدر: جمعت وحسبت من بيانات وزارة الزر اعة واستصلاح الأراضي، قطاع الثئون الاقتصادية، نشر ات الإحصاءات الزر اعية،

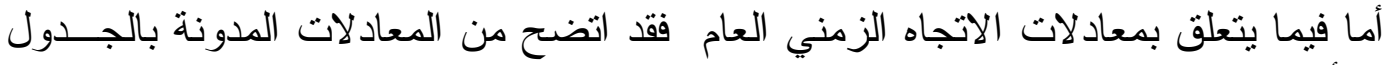

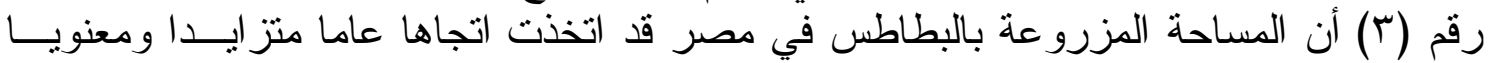

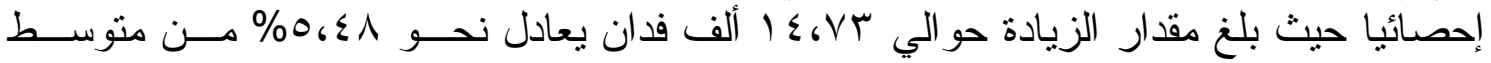

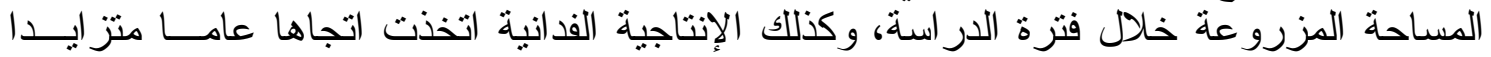

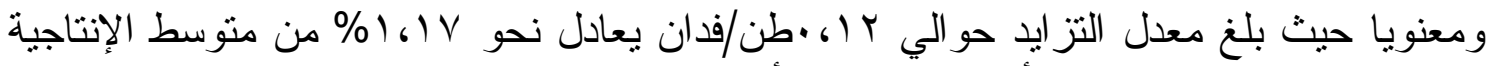

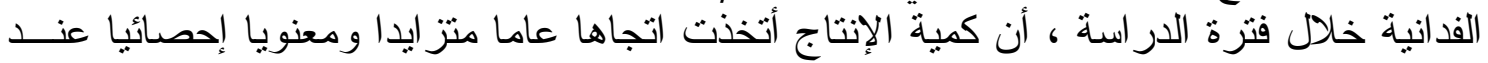

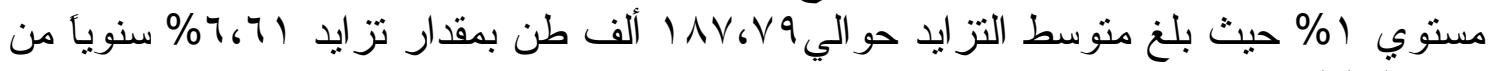

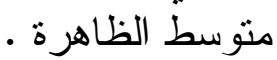

جدول رقم (ب): الاتجاهات الزمنية العامة لتطور إنتاج البطــاطس المــصرية خــلال القتــرة $(r \cdot 1 r-199 \mathrm{r})$

\begin{tabular}{|c|c|c|c|c|c|c|}
\hline التغير & المتوسط & $\mathbf{F}$ & $\mathbf{R}^{2}$ & $\mathbf{R}$ & النموذج المستخدم & المتغبر التابع \\
\hline 5.48 & 268.58 & 72.98 & 0.83 & 0.91 & $\hat{Y}=136.02+14.73 X_{i} * *$ & المزاحة \\
\hline 1.17 & 10.42 & 293.73 & 0.95 & 0.98 & 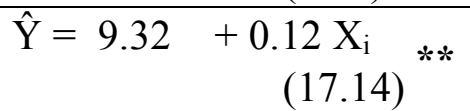 & الانتاجية \\
\hline 6.61 & 2841.05 & 100.31 & 0.87 & 0.93 & $\begin{array}{r}\hat{\mathrm{Y}}=1150.91+187.79 \mathrm{X}_{\mathrm{i}} \\
(10.02)^{* *}\end{array}$ & الانتاج الكلي \\
\hline
\end{tabular}

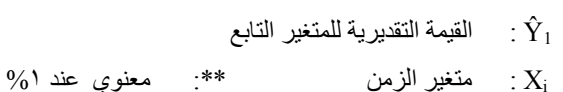

$$
\begin{aligned}
& \text { المصدر : جمعت وحسبت من بيانات الجدول رقم (r) }
\end{aligned}
$$

ثانيا : المعالم التصديرية للبطاطس المصرية:

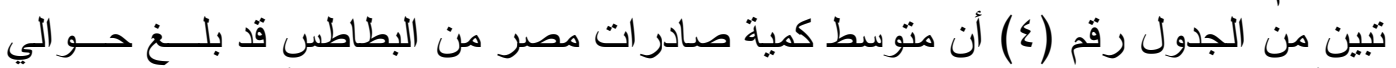

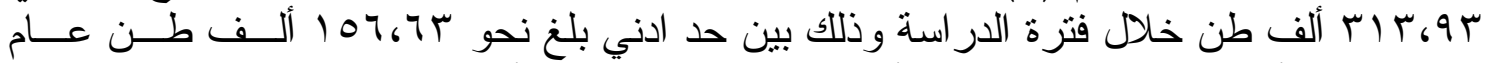

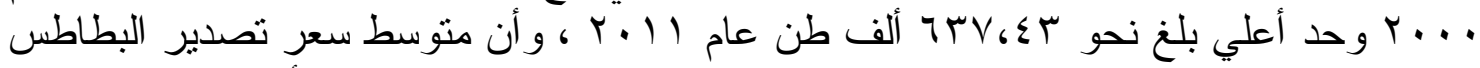

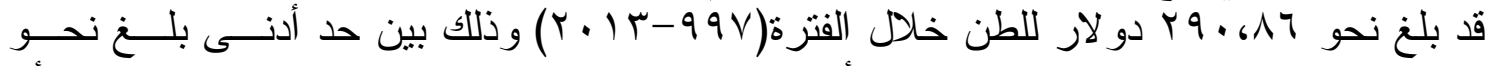

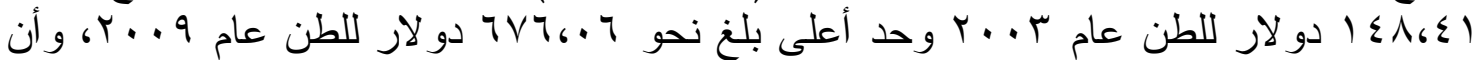

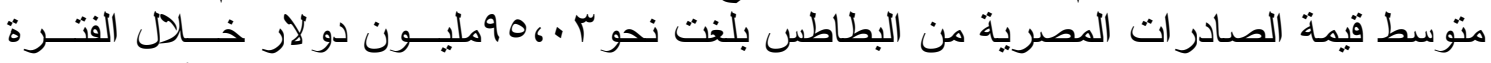

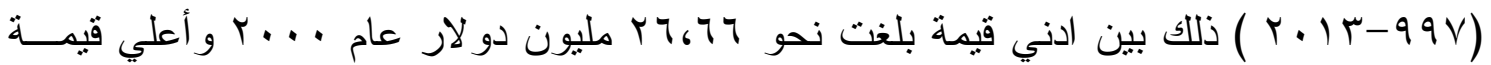

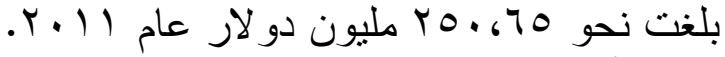

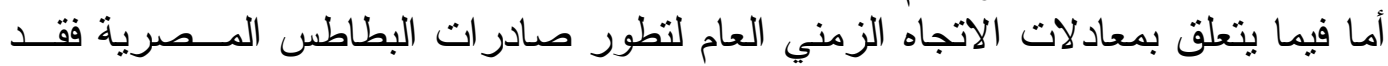

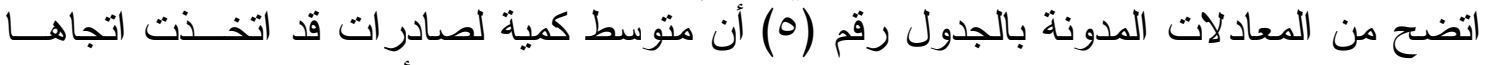

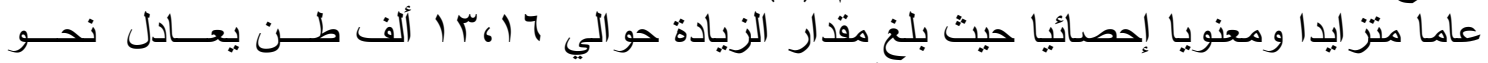

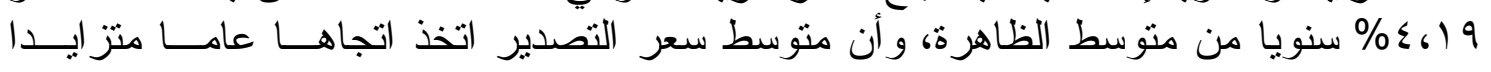

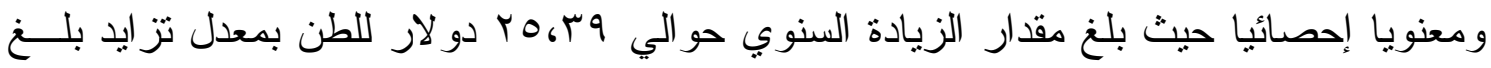




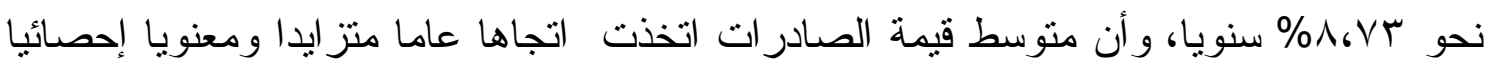

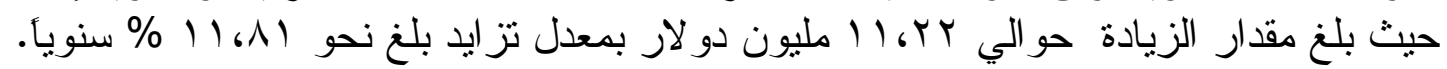




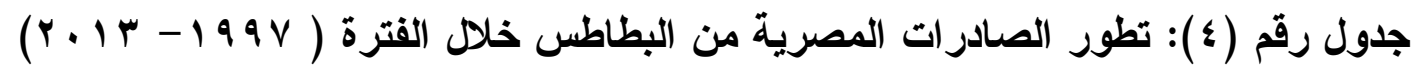

\begin{tabular}{|c|c|c|c|c|c|c|}
\hline الرقم القياسي & بالمليون & الرقم القياسي & متوسط السعر & القياسي الرقم \% & بالألف طن & البيان \\
\hline $1 \ldots$ & E1, YO & $1 \ldots$ & $1 V V_{6} \cdot 7$ & $1 \ldots$ & YTY.97 & $199 V$ \\
\hline $1 \cdot \varepsilon_{6} \vee \wedge$ & $\varepsilon T_{G} T Y$ & 1.7610 & 119619 & $199 \cdot V$ & YYA $6 \Sigma V$ & 1991 \\
\hline 111,09 & $\sum 7_{6} \cdot r$ & $1.16 \mathrm{Vr}$ & $1 \wedge .61 Y$ & $1.96 \mathrm{~V} 1$ & Y00,0V & 1999 \\
\hline$T \leq 67 T$ & YY.7T & 97.11 & 18.619 & $T V G T T$ & 107674 & $r \ldots$ \\
\hline$\left.V Y_{6}\right) T$ & r96. & $9.60 \mathrm{~V}$ & $17.6 \mathrm{TV}$ & $V 967 \%$ & $110,0$. & $r . .1$ \\
\hline I. T, & $\sum Y_{6} T Y$ & $1.569 \pi$ & $1106 \times 9$ & $91_{6} \leqslant 7$ & rYq.r人 & $r \ldots r$ \\
\hline 1.7 .09 & $\varepsilon r_{6} 9 V$ & Ar.Ar & $1 \leq \Lambda_{6} \leqslant 1$ & IYV6IN & Y976r9 & $r . . r$ \\
\hline $17 Y_{6} 0 \mathrm{~V}$ & $7 V_{6} \cdot 7$ & 99.40 & 170.91 & $17 r 675$ & rᄉI, & $r \cdot \varepsilon$ \\
\hline $1 \wedge V_{6} \vee \wedge$ & $V_{V} V_{6} \leqslant 7$ & 111.07 & $19 V_{6} 0 Y$ & $17 \Lambda_{6} 10$ & $r_{\left.9 r_{6}\right) \wedge}$ & $r . .0$ \\
\hline $10 \Lambda_{6} \cdot 1$ & 70.11 & $1 \cdots r V$ & IVV.OT & $10 V .09$ & TYV.IT & $r . . T$ \\
\hline Y7167. & 1.8691 & 107.19 & $r \vee 7_{69 .}$ & ITVGYA & 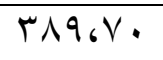 & $r \ldots r$ \\
\hline$r 90_{6} \leqslant V$ & 174,14 & $r r \varepsilon$ \& & $5 \Gamma 16)$ & $I_{1} Y_{6} \varepsilon r$ & rVA ${ }^{\prime}{ }^{\prime} q$ & $r \ldots \Lambda$ \\
\hline ror,0l & $1 \leq 0, \varepsilon 1$ & r人)،人 & $7 \vee 7_{6} .7$ & QY,Tr & $r 10_{6} \cdot 1$ & $r .99$ \\
\hline$\Gamma 196 V 7$ & $1 \pi 1.9$. & $r \leqslant \Lambda_{6} r_{0}$ & $\varepsilon r q_{6} V T$ & I YA.VT & r99.97 & r. I. \\
\hline $7.867 \leq$ & ro.670 & YYY $Y_{6} \cdot A$ & rqT,rr & TVTYA & $7 V_{6} \leq T$ & $r .11$ \\
\hline$r \cdot \Lambda_{6} V Y$ & IYVGO & YVTro. & $\varepsilon \wedge \varepsilon_{6} Y O$ & $11 r_{6} \wedge 9$ & Y7r.9A & $r \cdot 1 r$ \\
\hline$\leqslant 99610$ & $r .0,9$. & YVI6VT & $\Sigma \wedge \mid ، \backslash \wedge$ & I^r. & $\left\{Y V_{6} q 1\right.$ & $r \cdot 1 r$ \\
\hline- & 906.4 & - & rq. .А & - & rוr.9 & المتوسط \\
\hline
\end{tabular}

المصدر : جمعت وحسبت من بيانات United nation Statistics Division -Commodity trade Statistics database (www.comtrade.UN.Org)

جدول رقم (ه): الاتجاهات الزمنية العامة لتطور صادرات البطاطس المـصرية خــلا القتـــة $(r+1 r-199 \mathrm{~V})$

\begin{tabular}{|c|c|c|c|c|c|c|}
\hline معدل & المتوسط & $\mathbf{F}$ & $\mathbf{R}^{2}$ & $\mathbf{R}$ & شكل النموذج المستخدم & رقم المعادلة \\
\hline 4.19 & 313.93 & 6.84 & 0.31 & 0.56 & $\begin{array}{c}\hat{Y}=196.27+13.16 X_{i} \\
(2.61)^{* *}\end{array}$ & كمية الصادرات \\
\hline 8.73 & 290.86 & 25.29 & 0.63 & 0.79 & $\begin{array}{c}\hat{Y}=63.81+25.39 X_{i} \\
(5.03)^{* *}\end{array}$ & منوسط سعر التصدير \\
\hline 11.81 & 95.03 & 36.55 & 0.71 & 0.84 & $\begin{array}{c}\hat{\mathrm{Y}}=-5.31+11.22 \mathrm{X}_{\mathrm{i}} \\
(6.05)^{*}\end{array}$ & قتمة الصادر ات \\
\hline
\end{tabular}


ثالثاً : مؤشرات متعلقة بالقدرة التنافسية للبطاطس المصرية في الأسواق العالمية:

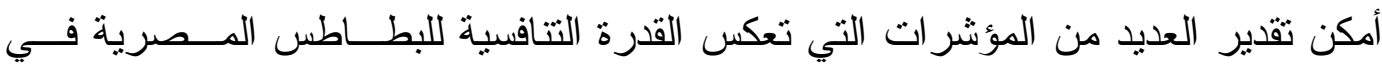
الأسو اق الخارجية طبقاً لما يلي: ألفئ 1 - كفاءة أداء العمليات التصديرية:

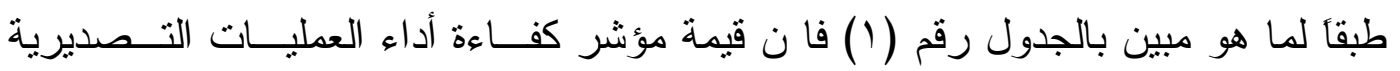

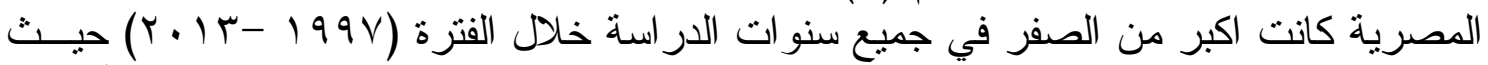

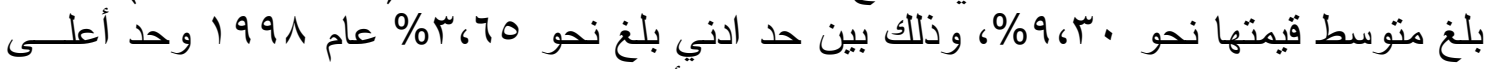

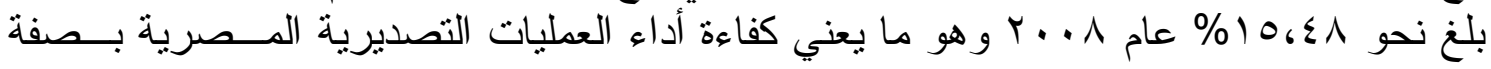

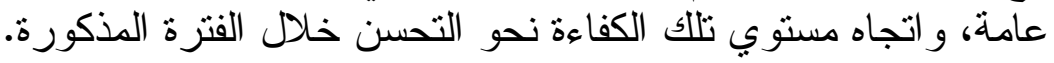

r - الميزة النسبية الظاهرية:

وبدر اسة الميزة النسبية الظاهرية للبطاطس المصرية تبين من الجدول رقدم الطية (7) إن مــصر

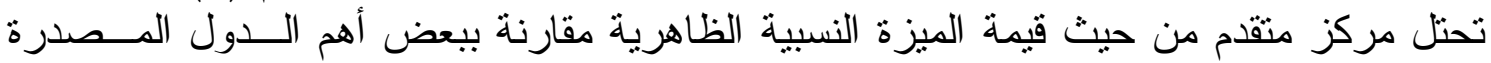

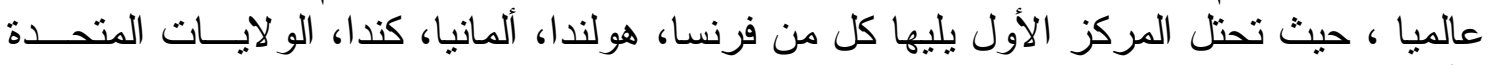

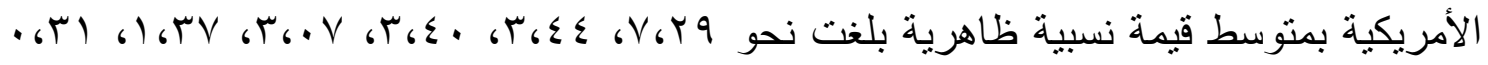

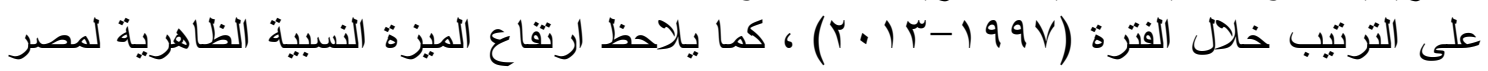

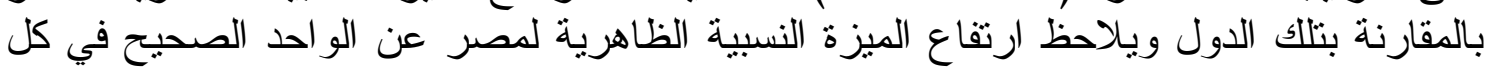

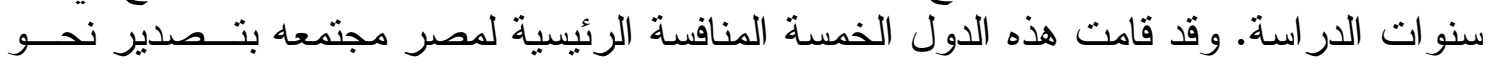

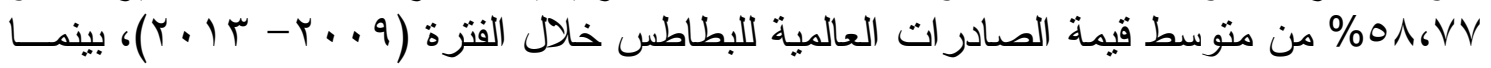
قامت مصر بتصدير ما يمثل نحو 01، ؛ \% من متوسط اجمالى قيمة صنادر ات البطاطس العالمية

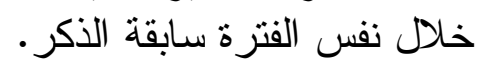
ب - التنافسية السعريه:

يتضح من الجدول رقم (V) تمتع صادر ات مصر من البطاطس بميزة نسبية سعريه أمسـام

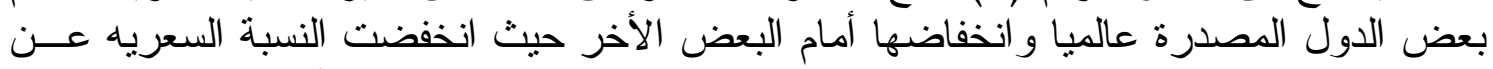

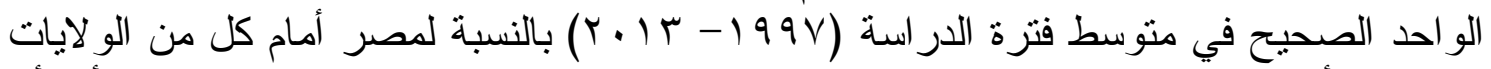

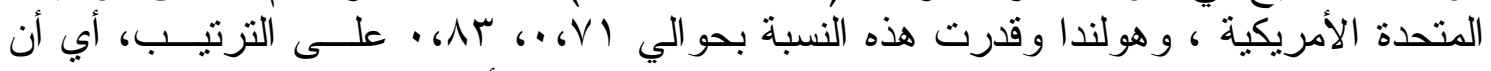

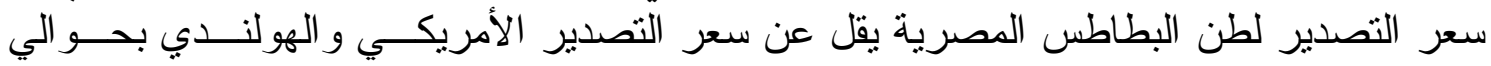

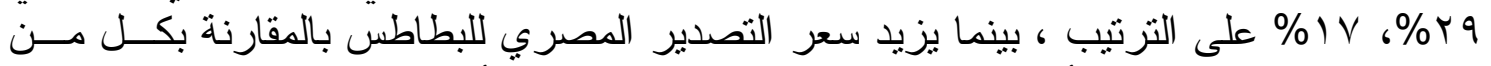

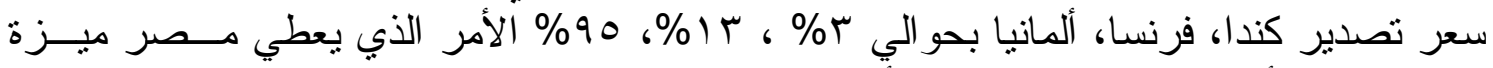

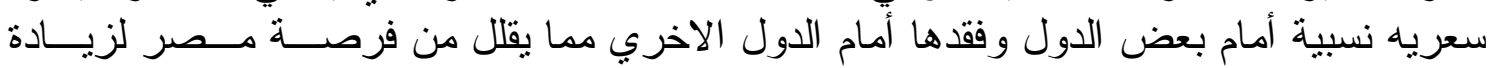
صادر اتها العالمية من البطاط لئس. 
جدول رقم (؟) : الميزة النسبية الظاهرية للبطاطس لبعض الدول المصدرة عالميا خلا الفترة $(r+1 r-199 v)$

\begin{tabular}{|c|c|c|c|c|c|c|}
\hline مصر & كندا & الألولايات المريكية & ألمانيا & فرنسا & هولندا & السنوات \\
\hline$I_{G} T^{\prime}$ & 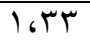 & .61 & T.TY & $r_{6} \cdot V$ & r.AY & $199 V$ \\
\hline$\left.V_{6}\right) \mathrm{V}$ & $167 \mathrm{~V}$ & $.6 \%$ & r.rT & $r_{6} \leqslant 9$ & $r_{6} \leqslant 7$ & 1991 \\
\hline 7.97 & $1, \leqslant \wedge$ & $.6 \mathrm{TA}$ & r.AT & T.rT & Y.9I & 1999 \\
\hline $0, T \leqslant$ & 167. & $.6 \mathrm{TV}$ & T.9T & $T_{6} \leqslant \varepsilon$ & $T_{617}$ & $r \ldots$ \\
\hline $0, Y$. & 1610 & . & $T_{6} V V$ & $r_{6} V_{T}$ & $r_{6} \varepsilon$. & $r \ldots l$ \\
\hline 0.70 & 1.91 & . ‘ $\varepsilon$. & r.Ar & Y.A. & r.71 & $r \ldots r$ \\
\hline $0, Y 0$ & 1671 & $.6 Y 7$ & $r_{6} \wedge 0$ & TrTo & T.9E & $r \ldots r$ \\
\hline 0.7. & 1619 & .619 & $\left.\Gamma_{6}\right) \varepsilon$ & r。A. & $r_{617}$ & $r \ldots \varepsilon$ \\
\hline$q_{6} \leqslant 1$ & $16 V Y$ & ד & r.91 & $r_{6} \leqslant r$ & T.7. & $r \ldots o$ \\
\hline $7, \leqslant V$ & $1, \%$ & $.6 \%$ & $r_{6} \wedge 1$ & r。A & $r_{6} \wedge \wedge$ & $r \ldots T$ \\
\hline$V .79$ & 1610 & $.6 T$ & r.97 & $\left.\varepsilon_{6}\right)$. & $r_{6} .$. & $r \ldots V$ \\
\hline$\Lambda_{6} \wedge \varepsilon$ & $16 r 0$ & $.6 Y 7$ & T.7T & $r_{6} \mu_{4}$ & $r_{6} . r$ & $r \ldots \lambda$ \\
\hline $0, \Sigma T$ & IGY & $.6 \mathrm{TA}$ & $r_{6} V_{0}$ & T.VT & $r_{6} r_{1}$ & $r .9$ \\
\hline$\varepsilon_{6} V Y$ & 1611 & $.6 Y 7$ & r.9r & $\varepsilon 61 \leqslant$ & $r_{6} \leqslant q$ & $r .1$. \\
\hline$\Lambda_{6} \wedge V$ & 1611 & $.6 \mathrm{TA}$ & r. & r.or & rov & $r .11$ \\
\hline$V_{G} T^{\prime}$ & 1611 & $.6 \mathrm{T \Lambda}$ & rov & $\varepsilon_{6}{ }_{T}$ & r.90 & $r .1 T$ \\
\hline $1165 \varepsilon$ & 161 & $.6 \leqslant 0$ & 7617 & 0.91 & $0, \leqslant 7$ & $r .1 T$ \\
\hline$V_{6} Y_{9}$ & $1, \mathrm{HV}$ & $.6 T$ & $r_{6} . V$ & $r_{6} \leqslant \varepsilon$ & r، & المتوسط \\
\hline
\end{tabular}

جدول رقم (V) : المركز التنافسي السعري للبطاطس المصرية مقارنة بيعض الدول المـصدرة

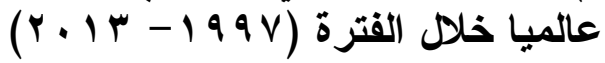

\begin{tabular}{|c|c|c|c|c|c|}
\hline سعر مصر بالنسبة & سعر مصر بالنسبة & سعر مصر بالنسبة & سعر مصر بالنسبة & سعر مصر بالنسبة & السنوات \\
\hline .69. & .670 & $16 \wedge 9$ & .697 & $.6 \wedge V$ & $199 V$ \\
\hline .694 & .674 & 1620 & .694 & $.6 \vee 7$ & 1991 \\
\hline. . $\wedge \mathrm{Y}$ & $.60 \mathrm{~V}$ & $1, \leqslant 0$ & $.6 \vee \leqslant$ & $.60 \mathrm{~V}$ & 1999 \\
\hline $.6 \wedge$. & .609 & $r_{6} \cdot \Lambda$ & $16 \cdot 4$ & .69. & $r \ldots$ \\
\hline $.6 V Y$ & $.60 Y$ & $r_{6.0}$ & .610 & $.6 \wedge 7$ & $r . .1$ \\
\hline $.6 \mathrm{~V}$. & $.6 \Omega \Lambda$ & 1.91 & .679 &.$، \wedge \Sigma$ & $r \ldots r$ \\
\hline .677 & $.6 \leqslant 0$ & $16 \mathrm{MA}$ & $.67 Y$ & .670 & $r . . r$ \\
\hline $.6 \wedge \mathrm{Y}$ & .601 & 161 & .609 & .674 & $r \ldots \varepsilon$ \\
\hline .610 & $.60 Y$ & $1.7 \mathrm{~V}$ & .694 &. . ‘AT & $r . .0$ \\
\hline .605 & $.6 \mathrm{T \Lambda}$ & $161 \mathrm{~V}$ & .677 & .60. & $r \ldots T$ \\
\hline $16 \ldots$ & .671 & $16 \leqslant 0$ &. . $A T$ & .609 & $r \cdots V$ \\
\hline .691 & $.6 \mathrm{VV}$ & Y.YT & 1.04 & .690 & $r \ldots \lambda$ \\
\hline 1,10 & 160. & $\varepsilon_{6} T V$ & $r_{69 .}$ & $161 \leq$ & $r . .9$ \\
\hline 1647 & $16 \cdot r$ & r. rN & 1677 & 16.0 & $r \cdot 1 \cdot$ \\
\hline 16.0 & $.6 \wedge 1$ & 1,00 & 1615 & $.6 \vee A$ & $r .11$ \\
\hline$r_{6} \varepsilon$. & 16.7 & T.VO & $16 \wedge 7$ & 1611 & $T \cdot I T$ \\
\hline $1,4 T$ & $16 \cdot 7$ & 1690 & 1641 & $.69 \varepsilon$ & $r \cdot 1 T$ \\
\hline 16.4 & $.6 \mathrm{VI}$ & 1690 & 1611 &. . & المتوسط \\
\hline
\end{tabular}


المصدر: جمعت وحسبت من بيانات الجدول رقم (Y (I)

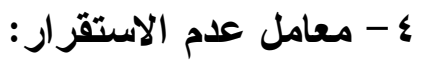

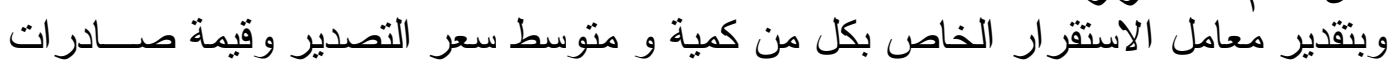

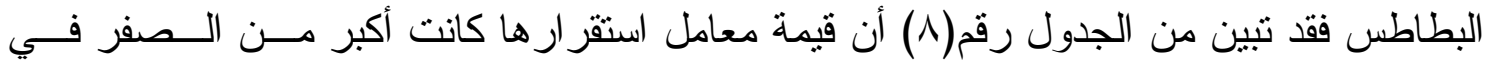

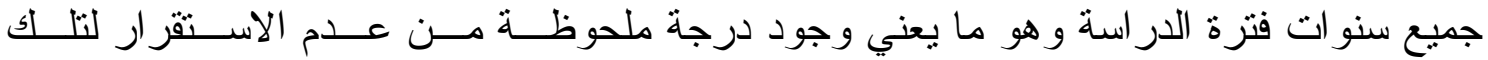

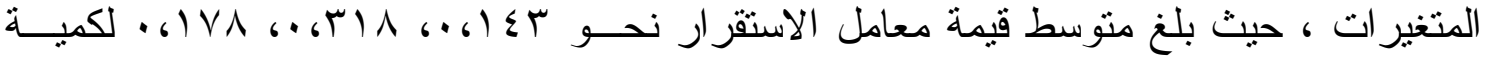

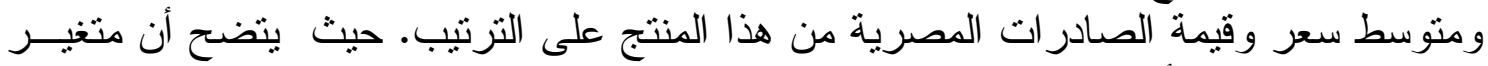
كمية التصدير هو الأكثر استقر ارَّ بين هذه المتغير ات.

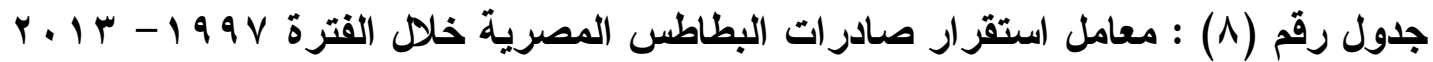

\begin{tabular}{|c|c|c|c|}
\hline القيمة & متوسط السعر & الكمية & السنوات \\
\hline$V .91$ & $.69 \leq$ & $.61 \varepsilon$ & $199 V$ \\
\hline 1621 & . 674 & $\cdot 6 \cdot 5$ & 1991 \\
\hline .679 & $.6 \mathrm{YA}$ & .61 & 1999 \\
\hline$(.6419)$ & $\cdot 6 \cdot Y$ & $(\cdot 6 \mathrm{*T})$ & r... \\
\hline$(\cdot ، \varepsilon \cdot)$ & $(.617)$ & $\left(.6 Y^{\prime}\right)$ & r.. \\
\hline$(\cdot .6 r)$ & $(.6) \leqslant)$ & $(.617)$ & $r \ldots r$ \\
\hline$(.649)$ & $\left(\cdot . \mathrm{r}^{\mathrm{N}}\right)$ & $.6 \cdot r$ & $r \ldots r$ \\
\hline$(\cdot G r \cdot)$ & $(\cdot . r \varepsilon)$ & $.6 Y V$ & Y.. \\
\hline$(.6) \wedge)$ & $(\cdot G r)$ & $.6 Y 0$ & r... \\
\hline$(.649)$ & $(\cdot 6 \leqslant \leqslant)$ & $.61 Y$ & $r \ldots r$ \\
\hline$(\cdot 6 \cdot 1)$ & $(.619)$ & $.61 \leq$ & $r \ldots V$ \\
\hline $.6 T V$ & .611 & $.6 \cdot V$ & $r \cdots \Lambda$ \\
\hline$\cdot 6 \cdot \varepsilon$ & $.6 \mathrm{VT}$ & $(\cdot, \leqslant Y)$ & $r \ldots q$ \\
\hline$(\cdot 6 / T)$ & .6 .7 & $\left(\cdot . Y_{1}\right)$ & $r \cdot 1$. \\
\hline .60$\}$ & $(.6) 1)$ & .671 & $r+11$ \\
\hline$(\cdot, r V)$ & $\cdot 6 \cdot \varepsilon$ & $(\cdot .6 \mathrm{MT})$ & T.IT \\
\hline .611 & $(\cdot \cdot \cdot r)$ & .6 .1 & $r .1 r$ \\
\hline .6$) \vee \wedge$ & $.6 \mathrm{TIN}$ & $.61 \leq \pi$ & الوسط الهندسي \\
\hline
\end{tabular}

المصدر: جمعت وحسبت من بيانات الجدول رقم (ع)

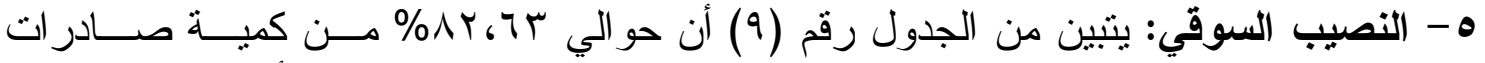

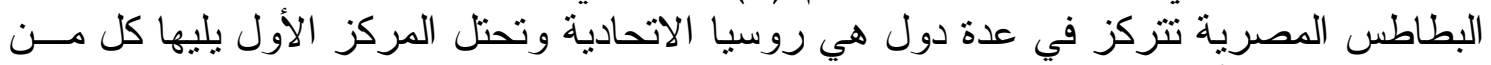

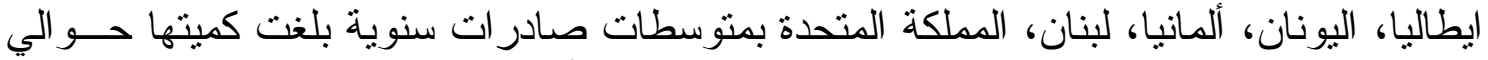
A (AV

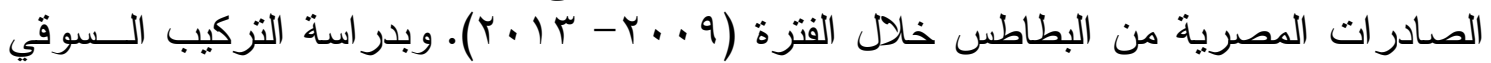

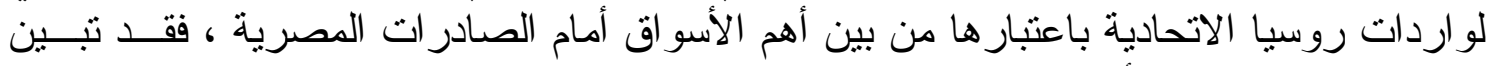

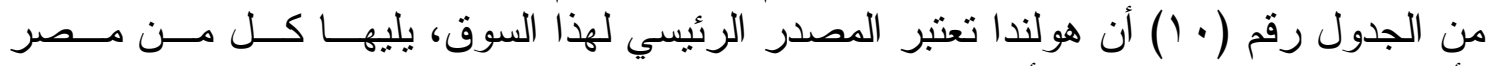

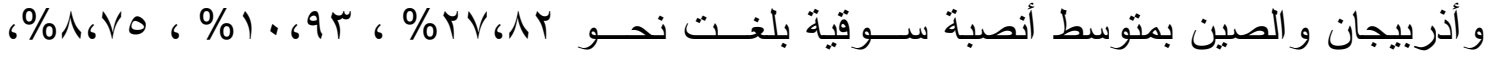

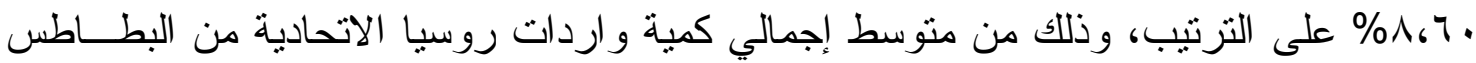

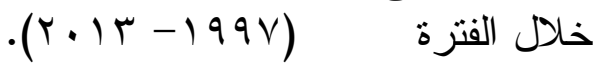




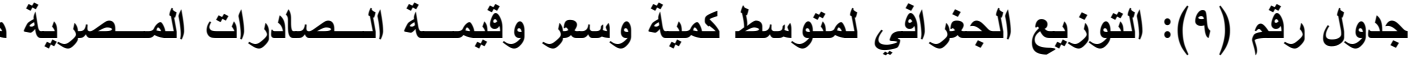

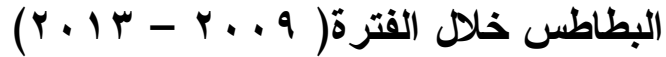

\begin{tabular}{|c|c|c|c|c|c|}
\hline \multicolumn{2}{|c|}{ القيمة بالمليون دولار } & \multirow{2}{*}{ متوسط السعر للطن } & \multicolumn{2}{|c|}{ الكمية بالألف طن } & \multirow{2}{*}{ البيان } \\
\hline$\%$ & متوسط القترة & & $\%$ & متوسط القترة & \\
\hline$r \Lambda_{6} V \cdot$ & $\varepsilon q_{6} \varepsilon r$ & $\leqslant 70,00$ & ع & $\left|Y_{6}\right| \wedge$ & روسبيا الاتحادية \\
\hline $1761 Y$ & $r V_{6} V V$ & $\varepsilon \vee 0_{6} \cdot 1$ & $1 \varepsilon_{6} \wedge V$ & $0 \leqslant ، \wedge r$ & ايطاليا \\
\hline $1 \leqslant 6$. & $r \leq 67 r$ & $\sum V Y_{6} V T$ & r. & $\varepsilon \Lambda_{6} 7 \vee$ & اليونان \\
\hline $9 . V V$ & 17.八 & $\varepsilon 70,1 T$ & 96.0 & 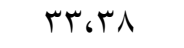 & ألماتيا \\
\hline$V_{6} 01$ & $1 r_{6} 9 \varepsilon$ & $\varepsilon V 1.9 Y$ & $V_{6} r_{9}$ & $r 7 . \wedge 9$ & لبنان \\
\hline $7 . r V$ & $1.69 V$ & $0 \leqslant 7619$ & $06 \cdot V$ & $\left|\Lambda_{6} \mathrm{~V}\right|$ & المملكة المتحدة \\
\hline IVGT & $r 9.79$ & $0 \cdot \cdots \leqslant \Lambda$ & IVGrV & $T \varepsilon_{6} \cdot T$ & دول أخري \\
\hline $1 \cdots 6$ & IVT6YE & $\left.\sum \wedge 16\right) \wedge$ & $1 \ldots 6$ & 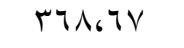 & إجمالي الصادر ات \\
\hline
\end{tabular}

المصدر : جمعت وحسبت من بيانات: United nation Statistics Division -Commodity trade Statistics database

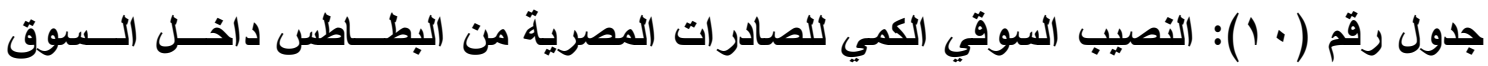

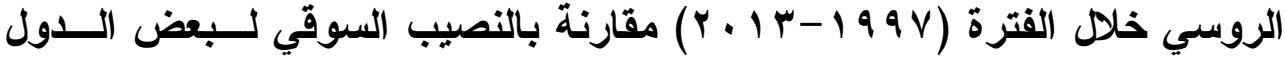

المنافسنة

\begin{tabular}{|c|c|c|c|c|c|c|c|c|c|}
\hline \multicolumn{2}{|c|}{ صادرات الصين } & \multicolumn{2}{|c|}{ صادرات أذربيجان } & \multicolumn{2}{|c|}{ صادر ات مصر } & \multicolumn{2}{|c|}{ صادرات هولندا } & \multirow{2}{*}{ بالألحف روسيات } & \multirow[b]{2}{*}{ السنوات } \\
\hline الكوقيب & بالألفية & الكوقيب & بالألفية & الكوقيب & بالألفية & الكبوقيب الكمي & بالألفية & & \\
\hline V.7 & 9611 & $.61 \mathrm{~V}$ & $.6 r$. & $\cdot 6 \cdots 0$ & .6 .1 & $r 96.0$ & $r \varepsilon 67 T$ & 119619 & $199 V$ \\
\hline 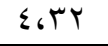 & 0.97 & $.61 \leq$ & $.6 r$. & $\cdot 6 . \varepsilon$ & .6 .0 & $\sum V_{6} \circ \mathrm{V}$ & $70 . V \leqslant$ & $\left|\mu_{1}\right| \Lambda$ & 1991 \\
\hline$\left.r_{6}\right)$. & $\varepsilon 6 r q$ & $\cdot 6 \cdots$ & $\cdot 6 \cdots$ & $\cdot 6 \cdots$ & $\cdot 6 \cdots$ & $1 \leq 694$ & $r .601$ & $r \cdot \varepsilon_{6} r \Lambda$ & 1999 \\
\hline 1.99 & 0,10 & $.6 \leqslant 0$ & $16 r 4$ & $\cdots \cdot r$ & $.6 \cdots 0$ & 7.610 & $17467 \varepsilon$ & Y7961人 & $r \ldots$ \\
\hline 7.7. & 7.71 & $.6 \mathrm{~V}$. & $.6 \mathrm{~V}$. & $\cdot 6 \cdots 1$ & $\cdot 6 \cdots 1$ & 17.79 & M. & $1.61 \mathrm{~V}$ & $r \ldots 1$ \\
\hline$r_{6} 01$ & $7 . V A$ & .671 & $161 \mathrm{~V}$ & $.6 \leqslant 9$ & .690 & Or.77 & $1.1 .0 \mathrm{~V}$ & 194.99 & $r \ldots r$ \\
\hline r.77 & $1 r_{6} \varepsilon 9$ & r.09 & $11 \%, 17$ & $\varepsilon 6 \vee \leqslant$ & $r V_{6} \cdot v$ & EI, Tा & $r .9690$ & $0 \cdot \Lambda_{6} \cdot 1$ & $r \ldots r$ \\
\hline 1.610 & $1 V_{6} 0$. & 18.01 & rA, ro & $1 r_{6} \cdot r$ & $196 \mathrm{rV}$ & 10.7 & rO, r. & 171645 & $r \ldots \varepsilon$ \\
\hline $11_{6} \wedge 9$ & OY,TI & $1 \cdot 6 \leqslant 1$ & 19619 & $1 r_{6} \varepsilon r$ & $0.60 \leqslant$ & 10,11 & 0961. & rVT،01 & $Y \ldots$ \\
\hline $11_{6} 10$ & 79.91 & $1 r_{6} 74$ & $\varepsilon 7_{6} \wedge \varepsilon$ & 19611 & $\vee \cdot ، \wedge \wedge$ & YY, & $\left.\wedge r_{6}\right) \varepsilon$ & $r v \cdot 69 r$ & $r \ldots q$ \\
\hline $11_{6} \times 9$ & $\sum 7, Y 9$ & $r \leqslant 670$ & $7 r_{6} .0$ & ro, 10 & 706.7 & תוT & $10.9 \varepsilon$ & YO1,VT & $r \cdot v$ \\
\hline 11641 & $7.6 Y \varepsilon$ & 10.97 & $10 . \mathrm{VV}$ & $111_{6} 9$. & $1.1 .0 \mathrm{~V}$ & rฯ,A & $1 \varepsilon \varepsilon 6 r \wedge$ & orv، $\leqslant q$ & $r . \Lambda$ \\
\hline 1. . & $\varepsilon .601$ & r r.os & 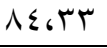 & 10.61 & $O \Lambda_{6} V V$ & rV.lT & $1.16 \leqslant V$ & $r V \leqslant 610$ & $r . . q$ \\
\hline $0_{6} T$. & TO,Tr & $96 \leqslant \leqslant$ & Tr.V. & 11,69 & V0.77 & $r \cdot 6 \leqslant r$ & $\left.r \cdot r_{6}\right)$ & Iフ & r. I. \\
\hline$\varepsilon_{6} \ldots$ & $O \Lambda_{6} V$. & 7.17 & 9.61 & $18.9 \leq$ & rาr. & Y1, ro & r & $1 \leq 77.4 T$ & $r .11$ \\
\hline 11.19 & 01.71 & $1 Y_{6}{ }^{\prime}$ & $0 \Lambda_{6} 7 \pi$ & $1 V_{6} 74$ & NI.TI & 11.04 & or. IV & $\varepsilon 71,1\}$ & $r .1 r$ \\
\hline Kr.17 & OV.TY & $1 r_{6} \cdot 1$ & or.As & rA, OV & $1 Y \Lambda_{6} \cdot 7$ & 11.04 & 01.74 & $\varepsilon \leqslant \Lambda_{6} Y$. & $r \cdot 1 r$ \\
\hline$\Lambda_{6} 7$. & M.人7 & A. Vo & $r q, r q$ & 1.695 & $0 \Lambda_{6} \times 1$ & rV.AT & 9967. & rq. & المتوسط \\
\hline
\end{tabular}

المصدر: جمعت وحسبت من بيانات (www.comtrade.UN.Org) 


\section{جدول رقم (11) : إجمالي قيمة الصادرات الزراعية بالمليــار دولار لأهــم الـــول المـصدرة}

للبطاطس :

\begin{tabular}{|c|c|c|c|c|c|c|c|}
\hline صصر مادر & صندا & 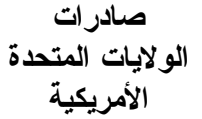 & صادادرات & فرنسا & هولندا & صادرات العالم & السنوات \\
\hline $.6 r 7$ & $V 679$ & TI.rT & 1690 & $\Lambda_{6} 9 \mathrm{r}$ & 961. & $1 \leqslant 7.71$ & $199 V$ \\
\hline $.60 Y$ & 7.77 & TV.)T & $\left.\varepsilon_{6}\right) V$ & $\Lambda_{6} \wedge \mu$ & 9617 & $1 \leqslant \varepsilon 6 r$. & 1991 \\
\hline .60 & $0 . \wedge 9$ & $r \varepsilon$ rAr & $\varepsilon_{6} \cdot \varepsilon$ & $\Lambda_{6} \wedge r$ & 961. & 117.97 & 1999 \\
\hline .601 & $7.1 T$ & YT.TE & r.人9 & $V_{6} V_{\varepsilon}$ & $961 T$ & 110610 & $r \ldots$ \\
\hline .604 & 7.19 & Y7.7. & $\varepsilon, 1 \leqslant$ & $V_{6},{ }^{\prime}$ & 96.0 & $1 \mathrm{VV}_{6} 10$ & $r \ldots 1$ \\
\hline .671 & $0,5 \leq$ & $r V_{6} 00$ & 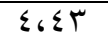 & V.9Y & 1.610 & $1 \leqslant Y_{6} V A$ & $r \cdot r$ \\
\hline $.6 \vee \wedge$ & 7.4. & Tr.Tq & $\varepsilon, V)$ & $96 \vee \varepsilon$ & $1 T_{601}$ & 179617 & $r \cdot r$ \\
\hline 1611 & $V_{6} 0 \leqslant$ & $r 0,59$ & $0, Y \leqslant$ & 1.60. & 106.7 & $19 T_{6} \mathrm{~V}$. & $r \cdots \varepsilon$ \\
\hline .694 & $V_{6} \leqslant \Lambda$ & $r \varepsilon_{6} r V$ & 7.199 & $1 \cdot 6 \leqslant 7$ & 10,57 & $r .0, r r$ & $r \ldots o$ \\
\hline. . \\
& $96 \leqslant \leqslant$ & $r \wedge_{6} \wedge \Lambda$ & $V_{6} Y V$ & $1.69 r$ & $\mid V_{6} T_{1}$ & YYVGVY & $r \ldots r$ \\
\hline 1641 & I Y.V. & 01617 & 1,07 & $1 r_{6} \varepsilon r$ & $r 161 T$ & Y人Y, & $r \cdot v$ \\
\hline$T_{6} \ldots$ & $1 \Lambda_{6} \cdot r$ & 77.99 & 116.4 & $1 V_{6} 7 \varepsilon$ & YM.AE & T07.71 & $r . \Lambda$ \\
\hline$r_{6} \wedge 1$ & 106.4 & $0 \leqslant 6199$ & 1.61 & $T_{6} T_{2}$ & Y1.01 & $\Gamma \Lambda_{6} \cdot \Lambda$ & $P .99$ \\
\hline$r_{6} \wedge 0$ & 10,01 & $T \Sigma G T \leq$ & $1.67 \varepsilon$ & $1 \leq 691$ & YY.00 & ryะ6). & $Y .1$. \\
\hline Y.VV & 196.7 & $V 7.01$ & IY,Tr & 19610 & $r v_{6} v_{q}$ & $\varepsilon \varepsilon \Lambda_{6} \vee r$ & 4.11 \\
\hline$T_{6} \leqslant \Lambda$ & $r .67 \Lambda$ & $V 7 . V Y$ & $116 \mathrm{AV}$ & $17 . A V$ & rV.OV & $\varepsilon 00_{6} \wedge r$ & $r .1 r$ \\
\hline$r_{6} \wedge I$ & Y1.11 & $V \varepsilon$ V(I) & $M M_{6} \varepsilon V$ & 19.09 & Y9.0V & $\leqslant 0 \leqslant 699$ & $r \cdot 1 r$ \\
\hline .61. & $1165 \varepsilon$ & $\leqslant 0, r$. & $V_{6} \leq 7$ & $M T_{6} \mid Y$ & $1 \Lambda_{6} \wedge 1$ & ro $\varepsilon_{6} \cdot 1$ & المتوسط \\
\hline
\end{tabular}

المصدر : جمعت وحسبت من بيانات (www.comtrade.UN.Org)

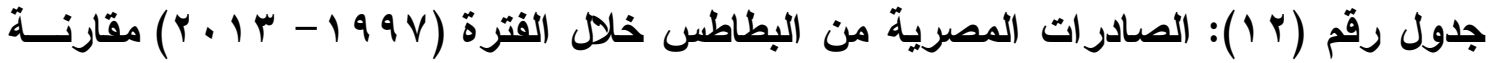
القيــة : بالمليــار دولار الكميـة :

بالألف طن

\begin{tabular}{|c|c|c|c|c|c|c|c|c|c|c|c|c|c|}
\hline & \multicolumn{2}{|l|}{ كندا } & \multicolumn{2}{|c|}{ الوالايات المتحدية } & \multicolumn{2}{|l|}{ ألمانيا } & \multicolumn{2}{|l|}{ فرنسا } & \multicolumn{2}{|l|}{ بولندا } & \multirow{2}{*}{ العادية } & \multirow{2}{*}{ السنو ات } \\
\hline الكمية & |القيمة & الكمية & |القيمة & الكمية & |القيمة & الكمية & | القيمة & الكمية & القيمة & الكمية & القيمة & & \\
\hline & & & & $\varepsilon, 0 Y$ & & & & & & & & (T) & \\
\hline & & & & $\varepsilon_{6} \cdot \varepsilon$ & .9 & & 11 & & ro & & & $67 \mathrm{~V}$ & 1 \\
\hline $1.0 \mathrm{~V}$ & 0 & 0 ○一9 & & Tq $\varepsilon_{6} .9$ & .699 & $1 Y 196$ & 10 & $12 \pi$, & $.6 \mathrm{YN}$ & 119.6 .4 & $.6 T \lambda$ & ،八 & 999 \\
\hline אד. &.$\Gamma$ & 9.07 & & I & $\ldots 9$ & To\&a. $\varepsilon$ & 611 & $11.6 \mathrm{rV}$ & 11 & $1 \leq \wedge \leq \varepsilon \varepsilon$. & $.6 Y \wedge$ & אי I, & $\cdots$ \\
\hline 60. & $\mu$ & & & $91.0 Y$ & .9 & $\varepsilon$ & $.61 \mathrm{~T}$ & $1116 \varepsilon \varepsilon$ & Y) & IVTrGIT & 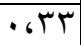 & $16 \leqslant V$ & $\cdots 1$ \\
\hline 6N & $6 \cdot \Sigma$ & Y.OY & $T$ & 0,11 & $.61 \mathrm{~T}$ & $r \Lambda_{1} 01$ & $.61 \leqslant$ & 01,09 & YO & 11996.0 & . $6 \leqslant Y$ & .09 & ar \\
\hline 649 & $\cdot \varepsilon$ & 1,09 & 11 & $.6 \mathrm{~V} \leqslant$ & .699 & 1 & $.61 \leqslant$ & $00,9 \leqslant$ & $.6 \% 0$ & IAVI,IT & 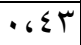 & $1 . \lambda 1$ & $\cdots r$ \\
\hline $\mid, T)$ & $\cdot v$ & 9 (६) & $1 \cdot$ & $\cdot r v \cdot$ & $\because 6 \mathrm{~V}$ & ITr., & $.61 \wedge$ & $9 r$ & $\mid \cdot(\varepsilon \Gamma$ & $1 \vee 97.94$ & .601 & $r_{6} \cdot \mathrm{V}$ & $\cdots \varepsilon$ \\
\hline Y.11 & .1 & \&Tr.0T & 611 & YA9,VT & .611 & 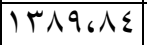 & .617 & $1 \leqslant 91,69$ & $.6 \mathrm{Tr}$ & 10.761. & ד דז6. & I.AY & $\ldots 0$ \\
\hline$V_{6} 1 T$ & $\cdot v$ & T\&,T & $1 \varepsilon$ & $\overline{A V} .9$. & $.61\}$ & $10 V 1679$ & $.6 Y \xi$ & IAYG,rV & $.6 \Sigma 9$ & $17 \varepsilon 1, \Lambda \varepsilon$ & .601 & Y.74 & $\cdots 7$ \\
\hline g.v. & 11 & .610 & IV & 906.7 & $.61 T$ & Y.OV & $.6 \% 9$ & 11.670 & $.67 \leqslant$ & $10 \vee 96 \varepsilon$. & $.6 \mathrm{~V} \varepsilon$ & GT & $\cdots v$ \\
\hline A.r9 & 17 & 19.19 & YI & r9.69) & .617 & 7.01 & $.6 \mathrm{TV}$ & 1950611 & .600 & $1 \leqslant V \leqslant V A$ & $.6 \mathrm{VV}$ & 6. & $\cdots 1$ \\
\hline $0, \cdot 1$ & 10 & $\varepsilon V_{6} V_{T}$ & IV & Tr..9T & .610 & $0,0 Y$ &. GYV & $r \cdot\{.6) T$ & 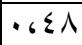 & 11.9 .49 & . . 74 & $r_{6.0}$ & $\ldots 9$ \\
\hline 9697 & 14 & ह9५. TV & IV & rᄉт, IN & $.61 \mathrm{~V}$ & $109 Y_{6} 0 Y$ & .64 & 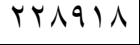 & .671 & $110 \mathrm{r} 69 \mathrm{~V}$ & $.6 \mathrm{VV}$ & r.OV & .1. \\
\hline$V_{6} \varepsilon Y$ & $.6 T_{0}$ & $71 \varepsilon_{6} 1 T$ & $r T$ & $\varepsilon 0 \varepsilon_{6} 9 \pi$ & $.6 T Y$ & 1017.TY & $\cdot$. $\varepsilon \cdot$ & 199.645 & .679 & $r \cdot \wedge 9, r)$ & 1.67 & $\varepsilon, 0 V$ & .11 \\
\hline 691 & $\pi$ & $\mathrm{V99}, \mathrm{VO}$ & 17 & $\varepsilon \varepsilon V_{6} 09$ & $r \cdot$ & $T V \varepsilon \varepsilon_{6} \ldots$ & . & $19 V \leqslant 899$ & .601 & $110 T_{6} \cdot V A$ & $.6 \mathrm{VT}$ & $\Gamma_{61 \Lambda}$ & $.1 Y$ \\
\hline & Y) & $\left.\varepsilon \neg \varepsilon_{6}\right) V$ & 11 & $\left.\sum V_{V} V_{6}\right) V$ &.$G Y Y$ & & $.00 \leqslant$ & 9.0 .99 & $.6 \mathrm{V7}$ & Y.TEGT & 16.0 & Y.97 & $r \cdot 1 r$ \\
\hline$\pi, 94$ & .610 & O.T.T & $.61 \leqslant$ & $r r 9610$ & .614 & & . & & . ، \&Y & & $.6 \mathrm{~V}$ & Y. $\varepsilon \varepsilon$ & لمتوسط \\
\hline
\end{tabular}


المصدر : جمعت وحسبت من بيانات United nation Statistics Division -Commodity trade Statistics database (www.comtrade.UN.Org)

(الملخص:

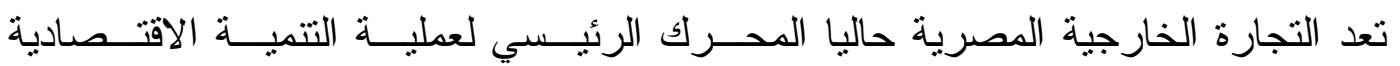

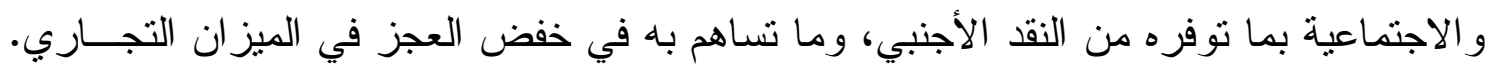

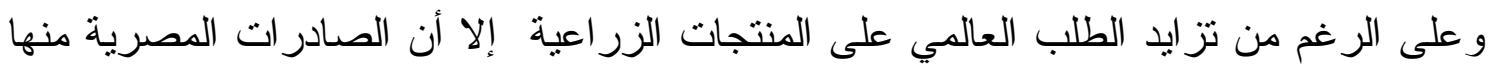

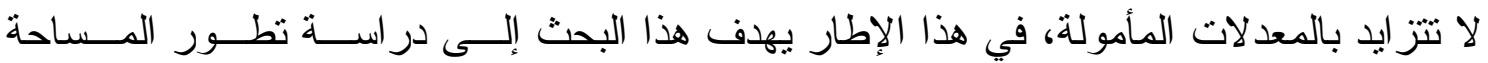

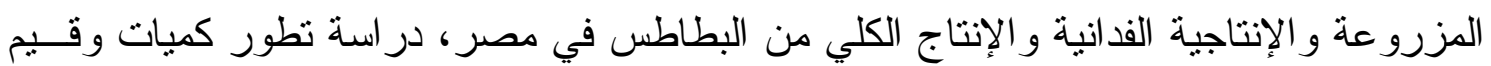

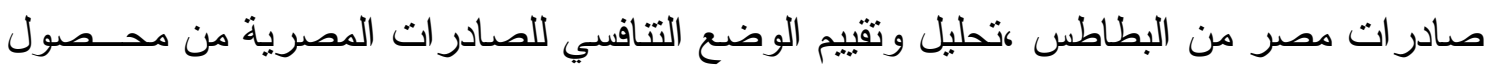

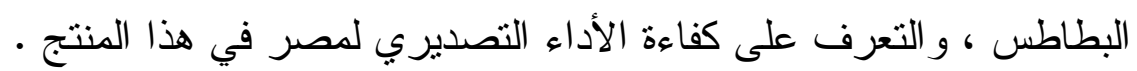
وقد توصلت الدر اسة إلي النتائج التالية:

1- أن متوسط المساحة المزروعة بالبطاطس في مصر قد بلغت نحو

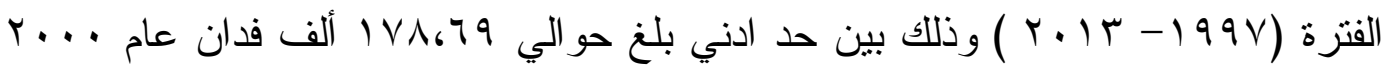

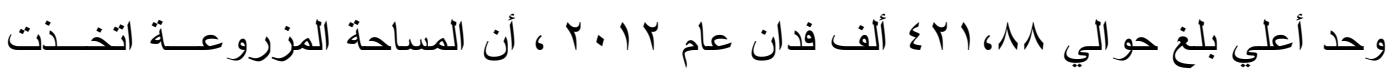

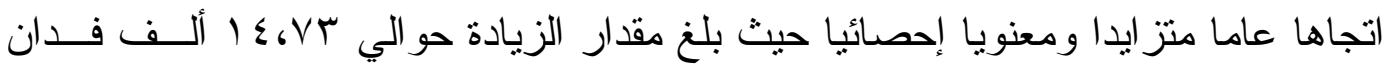

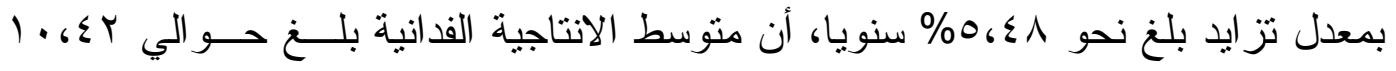

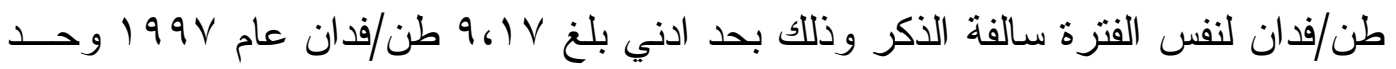

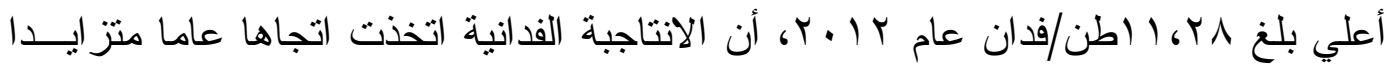

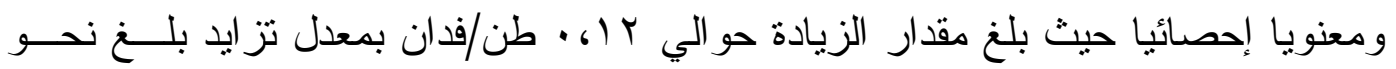

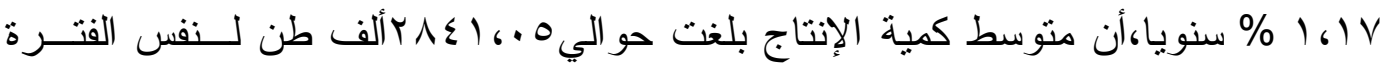

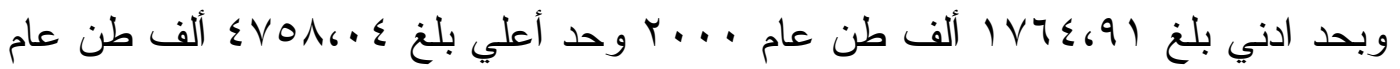

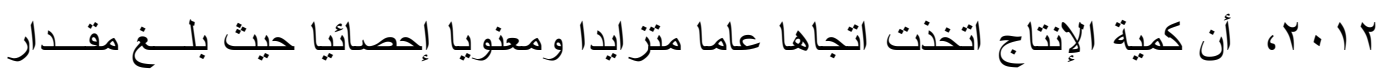

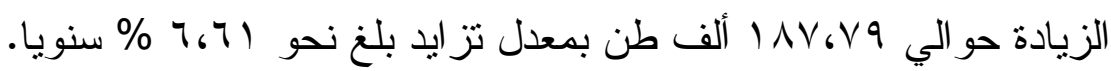

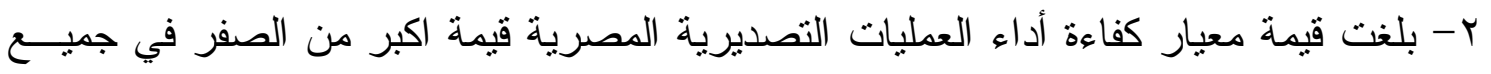

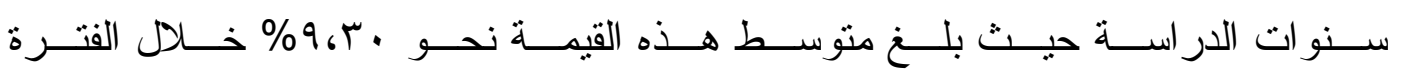

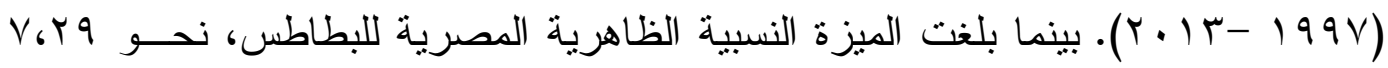

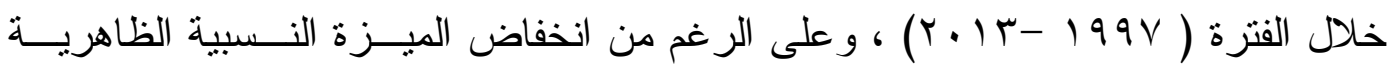

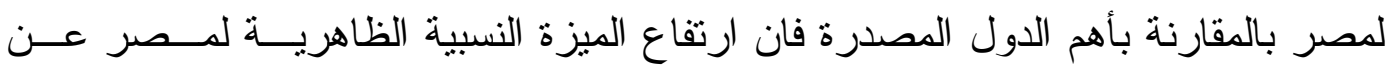
الو احد الصحيح خلال معظم سنو ات الدر اسة يدل على تمتع مصر بميزة نسبية ظاهرية.

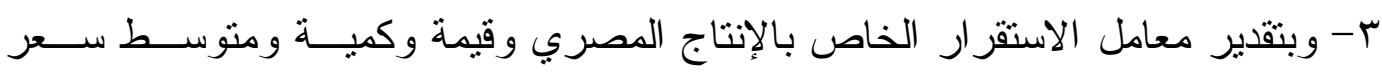

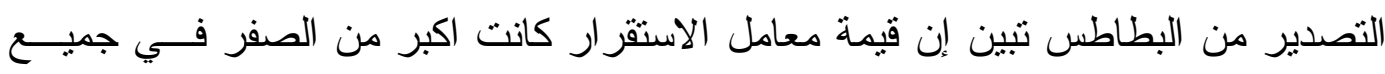

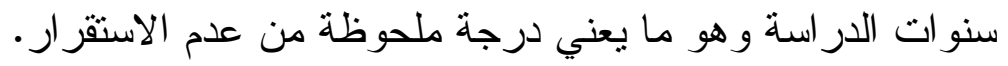

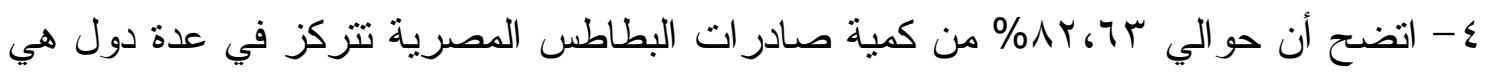

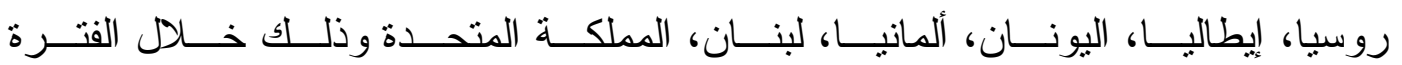

$$
\cdot(r \cdot 1 r-r \cdot .9)
$$


وبناء على ما نوصل إليه البحث من النتائج و المؤشر ات الخاصة بأهم معـايير التتافـسية

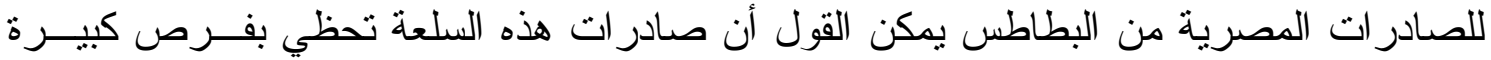

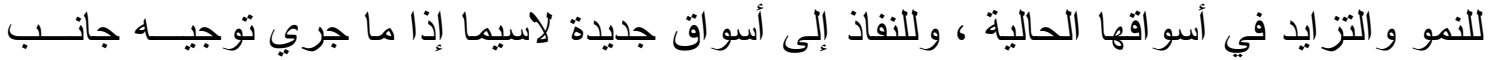

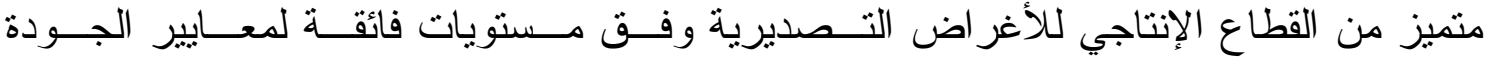
و المو اصفات مع المحافظة قدر الإمكان على الميزة النسبية السعرية الحالية التي تتمتع بها مصر

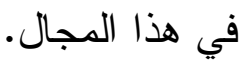
المر اجع: - اج

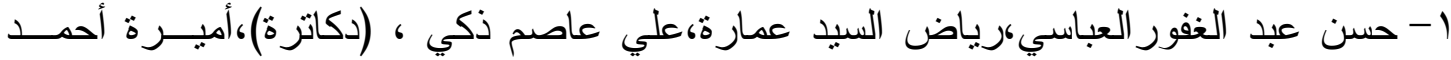

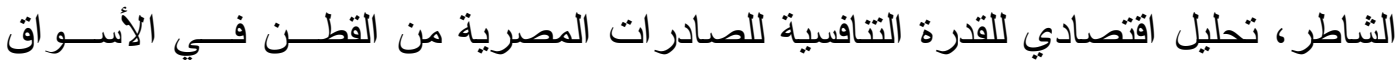

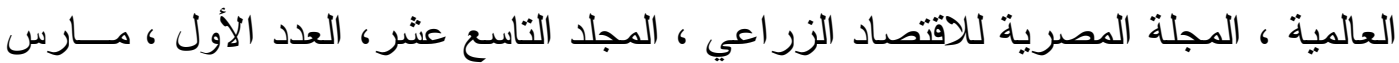
.r. . 9 ץ- ممتاز ناجي محمد السباعي ، محددات الطلب الخارجي على الــصادر ات المــصرية مــن

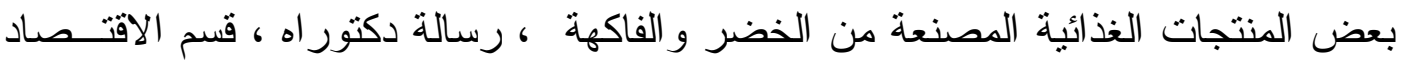
الزر اعي ، كلية الزر اعة ، جامعة عين شمس، 7 ، . . . .

3-United nation Statistics Division -Commodity trade Statistics database (www.comtrade.UN.Org) 


\title{
Production and Exports of Egyptian Potatoes and its Competitive Position in the World Markets
}

\author{
Omar N. Sultan ${ }^{2}$; Ahmed A. H. Mohamed ${ }^{1}$; Magdy M. Helal ${ }^{1}$ and \\ Mamdouh E. Mahmoud ${ }^{2}$ \\ ${ }^{1}$ Agricultural Economics - Faculty of Agriculture - Assiut University \\ ${ }^{2}$ Agricultural Research Center
}

\section{Summary:}

The Egyptian Foreign trade is currently the main Moving Factor for Economic and social development process by providing foreign exchange and contribute to the reduction of the trade deficit. Despite the growing global demand for agricultural products but exports are growing at rates expected in this context this research aims to study the evolution of the cultivated area and production for Feddan and potato production in Egypt, studying the evolution of quantities and values of Egypt's exports of potatoes, analysis and assessment of the competitive situation for potato exports, and identify efficient export performance of Egypt in this product

1-The most important results of the research include: the average area planted with potatoes in Egypt has reached about 268.58 thousand feddan during (1997-2013) and that the minimum approximately 178.69 thousand feddan in 2000 and the highest reached 421.88 thousand feddan in 2012, the cultivated area takes a general trend of increasing and statistically, with an average increase of about 14.73 thousand feddan at a rate increase of approximately $5.48 \%$ per annum.

2- The average yield was approximately 10.42 tons/feddan For the same period mentioned above with minimum reached 9.17 ton/feddan in 1997 and the highest reached 11.28 tons/Feddan in 2012, that average yield feddan take a general trend of increasing and statistically, with an average increase of about 0.12 tons/feddan rate increase of around $1.17 \%$ per annum, the average production of about 2841.05 thousand tons for the same period minimum reached 1764.91 tons in 2000 and the highest reached 4758.04 thousand tons in 2012, the amount of production take a general trend of increasing and statistically average More about 187.79 tons at a rate increase of about $6.61 \%$ per annum

3-As regards performance indicators competitive export results were measured as follows: value of standard performance of Egyptian export operations value greater than zero in all years of the study, with an average value around 9.30\% during the period (1997 - 2013). While the comparative advantage of the virtual Egyptian potatoes, about 7.29 during 1997-2013, although Egypt's virtual comparative advantage compared with the biggest exporters higher 
comparative advantage of Egypt from 1 during most of the years of study shows Egypt enjoyed a comparative advantage outward. And estimation of coefficient of stability of the Egyptian production, value and average export price and quantity of potatoes showed that the value of the coefficient of stability was greater than zero in all years of the study, which means a significant instability.

4-As it turns out that about $82.63 \%$ of exports of Egyptian potatoes are concentrated in several States are Russia, Italy, Greece, Germany, Lebanon, United Kingdom during the period (2009-2013). Based on the findings of the research results and indicators for the most competitive standards for exports of potatoes can be said that exports of these commodities have great opportunities for growth and the increase in the current markets, and access to new markets, especially if they were directed by the distinguished productive sector for export purposes in accordance with superior levels of quality standards and specifications while maintaining as much as possible on existing price comparative advantage enjoyed by Egypt in this area. 